\title{
DECAY OF CORRELATIONS IN ONE-DIMENSIONAL DYNAMICS
}

\author{
By HENK BRUIN, STEFANO LUZZATTO AND \\ SEBASTIAN VAN STRIEN
}

\begin{abstract}
We consider multimodal $C^{3}$ interval maps $f$ satisfying a summability condition on the derivatives $D_{n}$ along the critical orbits which implies the existence of an absolutely continuous $f$-invariant probability measure $\mu$. If $f$ is non-renormalizable, $\mu$ is mixing and we show that the speed of mixing (decay of correlations) is strongly related to the rate of growth of the sequence $\left(D_{n}\right)$ as $n \rightarrow \infty$. We also give sufficient conditions for $\mu$ to satisfy the Central Limit Theorem. This applies for example to the quadratic Fibonacci map which is shown to have subexponential decay of correlations.
\end{abstract}

๔ 2003 Éditions scientifiques et médicales Elsevier SAS

RÉSUMÉ. - Nous considérons des applications $C^{3}$ et multimodales de l'intervalle ayant des dérivées $D_{n}$ qui satisfont une condition de sommabilité le long de l'orbite critique, ceci entraînant l'existence d'une mesure de probabilité $\mu$ absolument continue par rapport à la mesure de Lebesgue. Si $f$ n'est pas renormalisable, $\mu$ est mélangeante et nous montrons que la décroissance de corrélation est fortement liée au rapport de croissance de la suite $\left(D_{n}\right)$ lorsque $n \rightarrow \infty$. Nous donnons également une condition suffisante pour que $\mu$ satisfasse au Théorème Central Limite. Ceci implique par exemple que l'application quadratique de Fibonacci possède une décroissance des corrélations sous-exponentielle.

○ 2003 Éditions scientifiques et médicales Elsevier SAS

\section{Introduction}

\subsection{Statement of results}

Let $f: I \rightarrow I$ be a $C^{3}$ interval or circle map with a finite critical set $\mathcal{C}$ and no stable or neutral periodic orbit. All critical points are assumed to have the same finite critical order $\ell \in(1, \infty)$. This means that for $c \in \mathcal{C}$, there exists a diffeomorphism $\varphi: \mathbb{R} \rightarrow \mathbb{R}$ fixing 0 such that for $x$ close to $c$,

$$
f(x)= \pm|\varphi(x-c)|^{\ell}+f(c),
$$

where the \pm may depend on $\operatorname{sgn}(x-c)$. For a critical point $c$, let

$$
D_{n}(c)=\left|\left(f^{n}\right)^{\prime}(f(c))\right|
$$

The aim of this paper is to prove the existence of an absolutely continuous invariant probability measure under sufficient growth conditions of $D_{n}(c)$ and to study its statistical properties (rate of mixing, Central Limit Theorem). In the proofs we will use distortion estimates valid for maps 
with negative Schwarzian derivative. By a result of Kozlovski [14] (generalized to the multimodal setting by van Strien and Vargas [24]), similar estimates hold if $f$ is $C^{3}$ and has no stable or neutral periodic orbit.

THEOREM 1 (Existence of invariant probability measures). - If $f$ satisfies

$$
\sum_{n} D_{n}^{-1 /(2 \ell-1)}(c)<\infty \quad \text { for each } c \in \mathcal{C}
$$

then there exists an $f$-invariant probability measure $\mu$ absolutely continuous with respect to Lebesgue measure (acip).

Moreover, as was shown in general in [12], $\operatorname{supp}(\mu)$ is an interval or cycle of intervals. In the unimodal case, Nowicki and van Strien [22] proved the same result under the assumption that $\sum_{n} D_{n}^{-1 / \ell}<\infty$. Theorem 1 is the first general existence theorem in the multimodal case, see [5]. Bruin and van Strien [8] later proved the following generalization: if $\sum_{n} D_{n}^{-1 / \ell_{\max }}(c)<\infty$ for all $c \in \mathcal{C}$ and $\ell_{\max }=\max \ell(c)$, then $f$ has an acip. In the holomorphic case, Przytycki proved that for every $\alpha$-conformal measure $\mu$ on $J$ (satisfying an additional assumption), assuming $D_{n}(c)$ grows exponentially, there exists an invariant probability measure which is absolutely continuous with respect to $\mu$, see [23]. Condition $(*)$ is equivalent to the following (see Lemma 2.1):

There exists a sequence $\left(\gamma_{n}\right), 0<\gamma_{n}<\frac{1}{2}$, such that $\sum_{n} \gamma_{n}<\infty$ and

$$
\sum_{n}\left[\gamma_{n}^{\ell-1} D_{n}(c)\right]^{-1 / \ell}<\infty \quad \text { for all } c \in \mathcal{C}
$$

We prefer to use this version of $(*)$, as the $\gamma_{i}$ play an important role in the binding method in the proof, and in the formulation of the other theorems. Let us also abbreviate

$$
b_{n}(c):=\left[\gamma_{n}^{\ell-1} D_{n}(c)\right]^{-1 / \ell}
$$

The measure $\mu$ need not be unique if $f$ is multimodal and not Lebesgue ergodic (unimodal maps with negative Schwarzian derivative are Lebesgue ergodic, [4]). Let $X$ be a closed $f$-invariant set of positive Lebesgue measure such that $X$ contains no smaller set with these properties. Misiurewicz [20] proved that $X$ contains a critical point. We do not know if $X$ can be a Cantor set (however, cf. [28]), but if $X$ supports the measure $\mu$ from Theorem 1, then $X$ has a non-empty interior. By a result of Ledrappier [15], $\mu$ is mixing if and only if $f: X \rightarrow X$ is not renormalizable, i.e. $X$ is not a cycle of intervals permuted by $f$. In this case it is natural to ask about the speed of mixing, quantified through the correlation function

$$
\mathcal{C}_{n}=\mathcal{C}_{n}(\varphi, \psi)=\left|\int\left(\varphi \circ f^{n}\right) \psi d \mu-\int \varphi d \mu \int \psi d \mu\right|,
$$

where $\varphi$ and $\psi$ are respectively bounded and Hölder continuous functions on $X$.

Write

$$
d_{n}(c):=\min _{i<n}\left[\gamma_{i} / D_{i}(c)\right]^{1 / \ell}\left|f^{i}(c)-\mathcal{C}\right| .
$$

Obviously, $d_{n}(c) \leqslant \gamma_{n-1} b_{n-1}(c)<b_{n-1}(c)$.

THEOREM 2 (Decay of correlations). - Let $f$ satisfy $(*)$ and let $\mu$ be an absolutely continuous invariant probability measure with support $\operatorname{supp}(\mu)$. If $f$ is not renormalizable on $\operatorname{supp}(\mu)$, then $(\operatorname{supp}(\mu), \mu, f)$ is mixing with the following rates: 
Polynomial case: If

$$
d_{n}(c) \leqslant C n^{-\alpha}
$$

for all $c \in \mathcal{C}$, some $\alpha>1$ and all $n \geqslant 1$, then for each

$$
\tilde{\alpha}<\alpha-1 .
$$

there exist $\widetilde{C}=\widetilde{C}(\varphi, \psi)>0$ such that

$$
\mathcal{C}_{n} \leqslant \widetilde{C} n^{-\tilde{\alpha}} \text { for all } n \geqslant 1 .
$$

Stretched exponential case: If

$$
b_{n}(c) \leqslant C \mathrm{e}^{-\beta n^{\alpha}}
$$

for all $c \in \mathcal{C}$, some $C, \beta>0, \alpha \in(0,1)$ and all $n \geqslant 1$, then for all $\tilde{\alpha} \in(0, \alpha)$ there exist $\widetilde{C}=\widetilde{C}(\varphi, \psi), \tilde{\beta}>0$ such that

$$
\mathcal{C}_{n} \leqslant \widetilde{C} \mathrm{e}^{-\tilde{\beta} n^{\tilde{\alpha}}} \text { for all } n \geqslant 1 .
$$

Exponential case: If

$$
b_{n}(c) \leqslant C \mathrm{e}^{-\beta n}
$$

for all $c \in \mathcal{C}$, some $C, \beta>0$ and $n \geqslant 1$, then there exist $\widetilde{C}=\widetilde{C}(\varphi, \psi), \tilde{\beta}>0$ such that

$$
\mathcal{C}_{n} \leqslant \widetilde{C} \mathrm{e}^{-\tilde{\beta} n} \text { for all } n \geqslant 1
$$

Notice that $d_{n}$ may decay much more rapidly than the terms of the series in condition (**). The formulation in terms of $d_{n}$ gives us an edge in the polynomial case. As an illustration, let us consider the case: $D_{n}(c) \geqslant C n^{\tau}, \tau>2 \ell-1$ for all $c \in \mathcal{C}$ and $n \geqslant 1$. Theorem 2 then tells us that $\mathcal{C}_{n} \leqslant \widetilde{C} n^{-\tilde{\tau}}$ for any $\tilde{\tau}<\frac{\tau-1}{\ell-1}-1$. Another use of the $d_{n}$ 's involves the quadratic Fibonacci map, see Corollary 1.1

If $\mu$ is an $f$-invariant probability measure, we say that the Central Limit Theorem holds if given a Hölder continuous function $\varphi$ which is not a coboundary $(\varphi \neq \psi \circ f-\psi$ for any $\psi$ ) there exists $\sigma>0$ such that for every interval $J \subset \mathbb{R}$,

$$
\mu\left\{x \in X: \frac{1}{\sqrt{n}} \sum_{j=0}^{n-1}\left(\varphi\left(f^{j}(x)\right)-\int \varphi d \mu\right) \in J\right\} \rightarrow \frac{1}{\sigma \sqrt{2 \pi}} \int_{J} \mathrm{e}^{-t^{2} / 2 \sigma^{2}} d t .
$$

This property is indicative of a certain regularity in the way Birkhoff averages of Hölder observable approach their expected asymptotic values.

THEOREM 3 (Central Limit Theorem). - Let $f$ satisfy $(*)$. If $f$ is not renormalizable and $d_{n}(c) \leqslant C n^{-\alpha}, \alpha>2$, for all $c \in \mathcal{C}$ and $n \geqslant 1$, then the measure $\mu$ of Theorem 1 satisfies the Central Limit Theorem.

The statements about decay of correlations and Central Limit Theorem in the unimodal exponential case were proved in $[13,30]$. As far as we know the results in all other cases are new. Most known examples of systems with strictly subexponential decay of correlations consist of maps which are uniformly expanding except for the presence of some neutral fixed point, see 
for example [16,31]. The situation here is more subtle as the cause for the loss of exponential estimates is not so localized.

In fact in the unimodal case, $D_{n} \geqslant C \mathrm{e}^{\beta n}$ if and only if $(X, \mu, f)$ has exponential decay of correlations [21].

It is interesting to apply the results to the Fibonacci maps, i.e. the conjugacy class of unimodal maps characterized by the property that the sequence of closest return times is exactly the Fibonacci sequence. Lyubich and Milnor [17] proved that in the quadratic case, the Fibonacci map satisfies Nowicki's and van Strien's summability condition. Here we show

COROLlaRY 1.1. - Let $f$ be a Fibonacci map with quadratic critical point. Then one has (faster than) polynomial decay of correlations and the central limit theorem holds.

Proof. - In fact, the estimates in [17, Section 5] show that condition (*) holds for e.g. $\gamma_{i}=0.01 \sqrt{1 / D_{i}}$, so $b_{n}=10 D_{n}^{-1 / 4}$. In [17, Lemma 5.9], it is shown that $\sum_{n} D_{n}^{-\alpha}<\infty$ for any $\alpha>0$, which leads to the existence proof of an acip. Theorems 2 and 3 also hold. Indeed, if $S_{r} \approx \gamma^{-r}$ is the $r$ th Fibonacci number (with $\gamma=(\sqrt{5}-1) / 2$ ), then

$$
\left|f^{S_{r}}(c)-c\right| \approx \mathrm{e}^{-\beta^{\prime} r^{2}} \approx S_{r}^{-\beta \log S_{r}}
$$

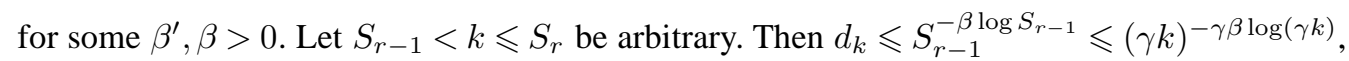
which decreases faster than any polynomial, but more slowly than what we call stretched exponentially. In particular, the Central Limit Theorem holds.

\subsection{Techniques and conjectures}

Our approach is to construct an induced Markov map and apply the result of L.-S. Young [32] which shows that the decay of correlations is tightly linked to the tail estimates of the inducing times. However, the construction of Markov induced maps (and the corresponding tower) is quite involved if the map has critical points. Expanding Markov induced maps have been constructed before, but only in the unimodal Collet-Eckmann setting tail estimates were undertaken. For our results, we need a new construction, which can be used for much weaker growth conditions on the orbits of multiple critical points, and indeed enables tail estimates of the inducing times. Apart from its use for estimating decay of correlations, towers were recently used by Collet [9] to describe return time statistics to small neighbourhoods. Indeed, combining our results (namely the tower structure with exponential tail behaviour, cf. Section 4.4) with Collet's paper, we can conclude that for all Collet-Eckmann multimodal maps with constant critical order, the quantity $\sup _{i \leqslant n}-\log \left|x-f^{i}(y)\right|$ satisfies Gumbel's law for $\mu$-a.e. $x$, see [9, Theorem 1.1] for details.

Since the growth of derivatives outside a neighbourhood of the critical set is exponential, one can argue that the tail is exponential for intervals which spend most of the time outside such neighbourhoods. Thus we need to concentrate particularly on intervals which fall inside critical neighbourhoods. One of the key ideas is to use a shadowing (or binding) argument to compare derivative growth for pieces of orbit to piece of critical orbit that they shadow. Binding arguments were developed by Jakobson [11] and Benedicks and Carleson [2], under strong growth assumptions $\left(D_{n} \geqslant \mathrm{e}^{\sqrt{n}}\right.$ or even $\left.D_{n} \geqslant \mathrm{e}^{\lambda n}\right)$ and slow recurrence of the critical point: $\left|f^{n}(c)-c\right| \geqslant \mathrm{e}^{\alpha n}$ for some small $\alpha$. This is the so-called basic assumption of [2]. Similar conditions were used in several papers concerned with dynamical and stability properties of various classes of one-dimensional maps. We mention [1] in particular where strong stochastic stability (for random perturbations) was proved, see also [25] where some similar conditions are introduced in the context of maps with completely flat critical points. 
We dispense with the slow recurrence assumption altogether, and introduce some new arguments in the construction:

- Our definition of binding period (see (4)) incorporates the recurrence pattern of the critical set. As a result, the partition of the space into intervals of constant induce time is not fixed in advance, as is the case in [2].

- In order to still count and measure the lengths of partition elements, we need intricate combinatorial counting arguments, which involves assigning itineraries to the partition elements, which indicate the "deepness" of the successive visits to a neighbourhood of the critical point.

- Our inducing time consist of three explicit parts: the first part is used to recover from the small derivatives near the critical set (thus achieving expansion); in the second intervals reach "large scale" and the third part is used to reach a prefixed interval.

In spite of the many differences, we believe that the construction is sufficiently robust as to justify

CONJECTURE 1. - Multimodal Collet-Eckmann maps are strongly stochastically stable.

Tsujii's result on weak stochastic stability [26] indicates in this direction. Possibly, the ColletEckmann condition itself can be replaced by a much weaker growth condition.

Let $\Lambda$ be a compact (forward) invariant set for a smooth map $f$ and $\mu$ be an $f$-invariant ergodic probability measure. The measure $\mu$ is called hyperbolic if all the Lyapunov exponents corresponding to $\mu$ are non-zero (recall that by Oseledec's Theorem, the Lyapunov exponents associated to a measure are well defined); it is called a physical measure if the set of $\mu$-generic points has positive probability with respect to the given reference (Lebesgue) measure. A nontrivial invariant set $\Lambda$ in general supports an infinite number of invariant measures some of which may be hyperbolic and some of which may not. At this point in the theory it is not completely clear how one could distinguish situations in which all invariant measure are hyperbolic and situations in which they are not. For the moment we suggest the following definition: we say that a compact invariant set $\Lambda$ is totally hyperbolic if all invariant measures with support on $\Lambda$ are hyperbolic. We conjecture that the presence of (singular) invariant measures with zero Lyapunov exponent (a natural generalization of the indifferent fixed point case), could be the main mechanism for slowing down of the mixing process and thus giving rise to only subexponential rates of decay of correlations.

CONJECTURE 2.-The map $f: \Lambda \rightarrow \Lambda$ exhibits exponential decay of correlations (with respect to every physical measure $\mu$ with support in $\Lambda$ ) if and only if $\Lambda$ is totally hyperbolic.

Conjecture 2 is true in the case of unimodal interval maps $f$ with negative Schwarzian derivative. Indeed, as was shown in [21], $f$ has exponential decay of correlations if and only if

$$
\lambda_{\text {per }}:=\inf \left\{\frac{1}{n} \log \left|\left(f^{n}\right)^{\prime}(p)\right| ; n \geqslant 1, p \text { is } n \text {-periodic }\right\}>0,
$$

and [6, Proposition 3.1] states that the Lyapunov exponent of any $f$-invariant measure is at least $\lambda_{\text {per }}$.

Different degrees of hyperbolicity might also influence the effect of small perturbations. Tsujii [27] showed that for generic one-parameter families unimodal maps satisfying a strong form of the Benedicks-Carleson conditions (and thus with exponential decay of correlations) are Lebesgue density points of similar maps.

CONJECTURE 3.-For generic one-parameter families, maps with exponential decay of correlations are Lebesgue density points of other maps with uniform exponential rates of decay 
of correlations. Maps with at least polynomial decay are Lebesgue density points of maps with (arbitrarily small) exponential decay.

\subsection{Overview of the paper}

Our strategy is to define a Markov return map $\widehat{f}=f^{R}: \Omega_{0} \rightarrow \Omega_{0}$ on a suitable neighbourhood of one of the critical points. We shall obtain estimates on the tail $\left|\left\{x \in \Omega_{0}: R>n\right\}\right|$ of the return times and apply the general framework of L.-S. Young [32] linking these estimates with bounds for the decay of correlation. The general philosophy is that intervals outside a neighbourhood $\Delta$ of the critical set grow exponentially fast (by a classical result of Misiurewicz [20], see [18] for a $C^{2}$ version) and therefore for these intervals during these times, the tail of the return times decays exponentially fast. Many intervals however fall into $\Delta$ before a good return to $\Omega_{0}$ occurs and are strongly contracted in the next iterate. We estimate the time it takes for them to recover their original size in terms of the derivatives along the critical orbits, which in turn provides bounds for the decay of the tail of the return time function.

In Section 2 we consider intervals in $\Delta$ and use a binding argument to obtain estimates for their growth in terms of the derivative along the appropriate critical orbit. As mentioned above, similar arguments have been applied before, notably by Jakobson [11] and Benedicks and Carleson [2], under stronger conditions on $D_{n}$ and on the recurrence of the critical orbit. Here we have generalized the argument to deal with slow derivative growth rates along the critical orbits and arbitrary recurrence patterns.

In Section 3 we consider an arbitrary interval $J \subset I$ and show that there exists a partition $\widehat{\mathcal{P}}$ of $J$ and a stopping time function $\hat{p}$ such that the images $f^{\hat{p}(\omega)}(\omega)$ are uniformly large for all $\omega \in \widehat{\mathcal{P}}$, i.e. almost every point of $J$ belongs to an interval which achieves large scale. We describe a combinatorial structure of $f^{\hat{p}}$ on $J$ which keeps track of the pattern of returns to $\Delta$ of each $\omega$. By combining this information with some analytic estimates on the size of elements with given combinatorics, we obtain key estimates on the size of the tail $\{x \in J: \hat{p}>n\}$ of the stopping time function $\hat{p}$. A variety of arguments is used here to deal with the various possible rates (polynomial, stretched exponential or exponential).

In Section 4 we show that once an interval has achieved large scale there is a fixed proportion of it which has a full return to the original interval $\Omega_{0}$ within a fixed number of iterates. It follows that the transition from large scale to full return occurs exponentially fast and does not significantly affect the tail estimates. We also state precisely the results of Young which we apply to our return map to obtain the conclusions of our theorems.

\section{Inducing to small scales}

We define a partition $\mathcal{P}$ of a critical neighbourhood $\Delta$ and a stopping time function $p$ such that the induced map $F=f^{p}$ on $\Delta$ is expanding. The images of partition elements are not uniformly large, i.e. $\inf \left\{\left|f^{p(\omega)}(\omega)\right|: \omega \in \mathcal{P}\right\}=0$, and therefore we call this inducing to small scales.

\subsection{Definitions and notation}

LEMma 2.1. - The conditions $(*)$ and $(* *)$ are equivalent.

Proof. - Condition $(*)$ implies $(* *)$ because if we take $\gamma_{n}$ so that $\gamma^{2 \ell-1}=D_{n}^{-1}$ then $\left[\gamma_{n}^{\ell-1} D_{n}(c)\right]^{-1 / \ell}=\gamma_{n}=D_{n}^{-1 /(2 \ell-1)}$, so the terms in each of the two sums in (**) are equal to each other and equal to those in $(*)$.

To see that $(* *)$ implies $(*)$ note that by the duality of $l^{p}$ and $l^{q}$ when $\frac{1}{p}+\frac{1}{q}=1, \sum a_{n}^{p}<\infty$, $\sum b_{n}^{q}<\infty$ implies $\sum a_{n} b_{n}<\infty$. Assume (**) holds and take $a_{n}^{p}=\gamma_{n}, b_{n}^{q}=\left[\gamma_{n}^{\ell-1} D_{n}(c)\right]^{-1 / \ell}$, 
$1 / q=\ell /(2 \ell-1)$ and therefore $1 / p=1-1 / q=(\ell-1) /(2 \ell-1)$. Then $\sum a_{n}^{p}$ and $\sum b_{n}^{q}$ are both finite and therefore $\sum a_{n} b_{n}<\infty$. But since $a_{n} b_{n}=\gamma_{n}^{\frac{1}{p}} \gamma_{n}^{-\frac{\ell-1}{\ell} \frac{1}{q}} D_{n}^{\frac{-1}{\ell q}}=D^{\frac{1}{2 \ell-1}}$, condition $(*)$ follows.

We use the symbol $\approx$ to indicate that two terms are equal up to a factor depending only on $f$. Because $\ell<\infty$,

$$
\left|f^{\prime}(x)\right| \approx|x-c|^{\ell-1}
$$

for all $x \in X$ close to $c$. Also there exists $\tau$ such that

$$
\frac{\left|f^{\prime}(x)-f^{\prime}(y)\right|}{\left|f^{\prime}(x)\right|} \leqslant \tau \frac{|x-y|}{|x-\mathcal{C}|},
$$

for all $x, y$ such that $|x-y| \leqslant \frac{1}{2} \max \{|x-\mathcal{C}|,|y-\mathcal{C}|\}$. Here $|x-\mathcal{C}|=\min \{|x-c| ; c \in \mathcal{C}\}$. Let $\Gamma=\exp \left(\tau \sum_{j=1}^{\infty} \frac{\gamma_{j}}{1-\gamma_{j}}\right)$.

For $x \in X$, let $c=c(x) \in \mathcal{C}$ be the critical point closest to $x$. This is well defined for $x$ sufficiently close to $\mathcal{C}$. Given a critical neighbourhood $\Delta$ of $\mathcal{C}$ we define the binding period as follows: If $x \in \Delta$, then

$$
p(x):=\max \left\{p:\left|f^{k}(x)-f^{k}(c)\right| \leqslant \gamma_{k}\left|f^{k}(c)-\mathcal{C}\right| \quad \forall k \leqslant p-1\right\},
$$

while $p(x):=0$ if $x \notin \Delta$. Clearly $p \rightarrow \infty$ monotonically as $x \rightarrow c(x)$. In order to choose the size of our critical neighbourhood $\Delta$ we need the following lemma. that

LEMmA 2.2. - Suppose that $G_{p} \geqslant 0$ and $\sum_{p} G_{p}<\infty$. Then for any $\zeta>0$ there exists $p_{0}$ such

$$
P=\sum_{s \geqslant 1} \sum_{\substack{\left.p_{1}, \ldots, p_{s}\right) \\ p_{i} \geqslant p_{0}}} \prod_{p_{i}} \zeta G_{p_{i}} \leqslant 1 .
$$

Proof. - Let $S_{0}=\sum_{p \geqslant p_{0}} \zeta G_{p}$. Then both $S_{0}$ and $S:=\sum_{s \geqslant 1} S_{0}^{s}$ tend to 0 as $p_{0} \rightarrow \infty$. Developing term by term we see that $P \leqslant S$. This proves the lemma.

LEMMA 2.3. - There exists $\kappa>0$ such that for all $\delta_{0}>0$, there exists $\delta \in\left(0, \delta_{0}\right)$ such that for $\Delta=\bigcup_{c}(c-\delta, c+\delta)$ and every $x$

$$
\left|\left(f^{n}\right)^{\prime}(x)\right| \geqslant \kappa \text { for } n=\min \left\{i \geqslant 0 ; f^{i}(x) \in \Delta\right\} .
$$

We call this property bounded backward contraction. In an earlier version of this paper [5], we had to state (BBC) as an assumption. For (symmetric) S-unimodal maps, (BBC) is well-known to hold, cf. [10], and recently the multimodal case it is proven in [7]. It is essential for (BBC) that all critical orders are the same, see the counterexamples in [7, Section 5].

Taking advantage of Lemma 2.2 and condition $(* *)$ we fix for the rest of the paper a critical neighbourhood $\Delta=\Delta_{\delta}=\bigcup_{c \in \mathcal{C}}(c-\delta, c+\delta)$ where $\delta>0$ is such that (BBC) holds and so small that

$$
\sum_{s \leqslant n} \sum_{\substack{\left(p_{1}, \ldots, p_{s}\right) \\ \sum_{i} p_{i} \leqslant n \\ p_{i} \geqslant p_{\delta}}} \prod_{p_{i}} \zeta\left(\gamma_{p_{i}}^{\ell-1} D_{p_{i}}(c)\right)^{1 / \ell} \leqslant 1
$$

for all $c \in \mathcal{C}, p_{\delta}:=p(c \pm \delta), \zeta=64 K_{0} / \kappa C_{0}, C_{0}$ the constant introduced in Lemma 2.5, and $K_{0}$ a fixed Koebe distortion constant, which turns out to be $\leqslant 16$. 
For $p \geqslant 0$ we let $I_{p}=\{x: p(x)=p\}$ denote the level sets of the function $p$. Let $\mathcal{P}$ denote the corresponding partition of $X$. Note that since $p(x) \equiv 0$ outside $\Delta, I_{0}=X \backslash \Delta$ is the "zeroth" partition element. Notice that $I_{p}$ can be empty for some values of $p$, and that it has at most $2 \# \mathcal{C}$ components. Define $F: X \rightarrow X$ by letting $F(x)=f^{p(x)}(x)$ for $x \in \Delta$ and $F(x)=f(x)$ for $x \in X \backslash \Delta$.

\subsection{Expansion estimates}

We have two main expansion estimates.

LEMMA 2.4 (Derivative growth for pieces of orbit outside $\Delta$ ). - There exist constants $C_{\delta}>0$ and $\lambda_{\delta}>0$ such that for every piece of orbit $\left\{f^{i}(x)\right\}_{i=0}^{k-1}$ lying completely outside $\Delta$ we have

$$
\left|\left(f^{k}\right)^{\prime}(x)\right| \geqslant C_{\delta} \mathrm{e}^{\lambda_{\delta} k} .
$$

If moreover $f^{k}(x) \in \Delta$, then

$$
\left|\left(f^{k}\right)^{\prime}(x)\right| \geqslant \max \left\{\kappa, C_{\delta} \mathrm{e}^{\lambda_{\delta} k}\right\}
$$

Notice that the first estimate clearly implies the second if $k$ is large. The second however is extremely useful when considering small values of $k$.

Proof. - The first estimate is well known for maps with negative Schwarzian derivative, and also for maps without periodic attractors or neutral orbits (Mañé's result), see Chapter II in [19]. So this covers our case. The second statement follows from (BBC).

The following expansion bound will be of importance. Let

$$
F_{p}^{\prime}(c):=\min \left\{\left|\left(f^{p}\right)^{\prime}(x)\right| ; x \in I_{p} \cap(c-\delta, c+\delta)\right\} .
$$

LEMMA 2.5 (Derivative growth for pieces of orbit starting in $\Delta$ ). - There exists $C_{0}>0$ (independently of $\delta$ and hence $\Delta$ ) such that for every $c \in \mathcal{C}$ and $p \geqslant p_{\delta}$ with $I_{p} \neq \emptyset$ we have

$$
F_{p}^{\prime}(c) \geqslant C_{0}\left[\gamma_{p}^{\ell-1} D_{p}(c)\right]^{1 / \ell}
$$

In the sequel, we will write $F_{p}^{\prime}$ instead of $F_{p}^{\prime}(c)$ when no confusion can arise. We shall need an intermediate result for the proof.

LEMMA 2.6. - For $x \in \Delta$ we have

$$
\frac{\left|\left(f^{i}\right)^{\prime}(y)\right|}{\left|\left(f^{i}\right)^{\prime}(z)\right|} \leqslant \Gamma \quad \text { for all } y, z \in[f(x), f(c)] \text { and all } i \leqslant p(x)-1 .
$$

Remark. - In Section 3.1 we will use this estimate on a slightly bigger interval than $[f(x), f(c)]$, but this does not seriously affect the estimates.

Proof. - Letting $y_{j}=f^{j}(y)$ and $z_{j}=f^{j}(z)$ for $j \geqslant 0$ we have by the chain rule

$$
\left|\frac{\left(f^{i}\right)^{\prime}(y)}{\left(f^{i}\right)^{\prime}(z)}\right|=\prod_{j=0}^{i-1}\left|\frac{f^{\prime}\left(y_{j}\right)}{f^{\prime}\left(z_{j}\right)}\right|=\prod_{j=0}^{i-1}\left(1+\frac{\left|f^{\prime}\left(z_{j}\right)-f^{\prime}\left(y_{j}\right)\right|}{\left|f^{\prime}\left(y_{j}\right)\right|}\right) .
$$


By (3),

$$
\left|f^{\prime}\left(z_{j}\right)-f^{\prime}\left(y_{j}\right)\right| /\left|f^{\prime}\left(y_{j}\right)\right| \leqslant \tau\left|z_{j}-y_{j}\right| /\left|y_{j}-\mathcal{C}\right|
$$

and so, using the elementary fact that $\log (1+x) \leqslant x$ for all $x>0$ we get

$$
\log \left|\frac{\left(f^{i}\right)^{\prime}(y)}{\left(f^{i}\right)^{\prime}(z)}\right| \leqslant \sum_{j=0}^{i-1} \log \left(1+\tau \frac{\left|z_{j}-y_{j}\right|}{\left|y_{j}-\mathcal{C}\right|}\right) \leqslant \tau \sum_{j=0}^{i-1}\left(\frac{\left|z_{j}-y_{j}\right|}{\left|y_{j}-\mathcal{C}\right|}\right)
$$

By definition of $p$ we have

$$
\left|z_{j}-y_{j}\right| \leqslant\left|f^{j+1}(x)-f^{j+1}(c)\right| \leqslant \gamma_{j+1}\left|f^{j+1}(c)-\mathcal{C}\right|
$$

and

$$
\left|y_{j}-\mathcal{C}\right| \geqslant\left(1-\gamma_{j+1}\right)\left|f^{j+1}(c)-\mathcal{C}\right| .
$$

Here $c$ is again the critical point closest to $x$. Substituting these inequalities into the last formula yields the desired statement.

Proof of Lemma 2.5. - Let $c$ be the critical point closest to $x$. By Lemma 2.6 and the fact that

$$
\left|f^{\prime}(x)\right| \approx|x-c|^{\ell-1} \approx|f(x)-f(c)|^{(\ell-1) / \ell}
$$

we have

$$
\left|\left(f^{p}\right)^{\prime}(x)\right| \geqslant \frac{\left|f^{\prime}(x)\right| D_{p-1}(c)}{\Gamma} \approx \frac{|f(x)-f(c)|^{(\ell-1) / \ell} D_{p-1}(c)}{\Gamma} .
$$

By the Mean Value Theorem, the definition of $p$ and the distortion estimate in Lemma 2.6 we have

$$
\Gamma D_{p-1}|f(x)-f(c)| \geqslant\left|f^{p}(x)-f^{p}(c)\right| \geqslant \gamma_{p}\left|f^{p}(c)-\mathcal{C}\right|
$$

and therefore

$$
|f(x)-f(c)| \geqslant \frac{\gamma_{p}\left|f^{p}(c)-\mathcal{C}\right|}{\Gamma D_{p-1}(c)} .
$$

Substituting (8) into (7) gives

$$
\left|\left(f^{p}\right)^{\prime}(x)\right| \geqslant \mathcal{O}\left(\Gamma^{-2+1 / \ell}\right) \gamma_{p}^{(\ell-1) / \ell} D_{p-1}(c)^{1 / \ell}\left|f^{p}(c)-\mathcal{C}\right|^{(\ell-1) / \ell} .
$$

Because

$$
\left|f^{\prime}\left(f^{p}(c)\right)\right| \approx\left|f^{p}(c)-\mathcal{C}\right|^{\ell-1}
$$

the Chain Rule gives

$$
\left|\left(f^{p}\right)^{\prime}(x)\right| \geqslant \mathcal{O}\left(\Gamma^{-2+1 / \ell}\right) \gamma_{p}^{(\ell-1) / \ell} D_{p}(c)^{1 / \ell}
$$

This proves the lemma. 


\section{Inducing to large scales}

The main result of this section is the following

Proposition 3.1. - Suppose that $f$ satisfies $(*)$. Then there exists $\delta^{\prime}>0$ such that for all $\delta^{\prime \prime}>0$ the following properties hold. For an arbitrary interval $J \subset X$ with $|J| \geqslant \delta^{\prime \prime}$ there exist a partition $\widehat{\mathcal{P}}$ of $J(\bmod 0)$ and a stopping time function $\hat{p}: \widehat{\mathcal{P}} \rightarrow \mathbb{N}$ such that for all $\omega \in \widehat{\mathcal{P}}, \widehat{F}\left|\omega:=f^{\hat{p}(\omega)}\right| \omega$ is a diffeomorphism with uniformly bounded distortion and $|\widehat{F}(\omega)|=\left|f^{\hat{p}(\omega)}(\omega)\right| \geqslant \delta^{\prime}$. Moreover the following estimates hold:

Summable case: Under no conditions on $d_{n}(c)$ other than those which stem from $(*)$

$$
\sum_{n}|\{\hat{p}>n \mid J\}|<\infty
$$

Polynomial case: If $d_{n}(c) \leqslant C n^{-\alpha}$ for all $c \in \mathcal{C}$ and $n \geqslant 1$, then there exists $C>0$ such that

$$
|\{\hat{p}>n \mid J\}| \leqslant \widehat{C} n^{-\alpha}
$$

Stretched exponential case: If $b_{n}(c) \leqslant C \mathrm{e}^{-\beta n^{\alpha}}, \alpha \in(0,1), \beta>0$ for all $c \in \mathcal{C}$ and $n \geqslant 1$, then for each $\hat{\alpha} \in(0, \alpha)$ there exist $\hat{\beta}, \widehat{C}>0$ such that

$$
|\{\hat{p}>n \mid J\}| \leqslant \widehat{C} \mathrm{e}^{-\hat{\beta} n^{\hat{\alpha}}} .
$$

Exponential case: If $b_{n}(c) \leqslant C \mathrm{e}^{-\beta n}, \beta>0$ for all $c \in \mathcal{C}$ and $n \geqslant 1$, then there exist $\hat{\beta}, \widehat{C}>0$ such that

$$
|\{\hat{p}>n \mid J\}| \leqslant \widehat{C} \mathrm{e}^{-\hat{\beta} n} .
$$

Let us try to clarify the role of the constants in this proposition, and their interdependence. In the previous section we have fixed $\delta$. By the Contraction Principle (see e.g. [19, Section IV.5]), there exists $\delta^{\prime}$ such that for each component $W$ of $\Delta \backslash \mathcal{C}$ and each $n \geqslant 0,\left|f^{n}(W)\right| \geqslant \delta^{\prime}$. This is the $\delta^{\prime}$ of Proposition 3.1.

The expression $|\{\hat{p}>n \mid J\}|$ denotes the conditional probability $|\{x \in J ; \hat{p}(x)>n\}| /|J|$. In Section 3.1 we define and describe the combinatorics of the partition $\widehat{\mathcal{P}}$ of $J$ and the stopping time $\hat{p}$. In Section 3.2 we prove some key estimates on the size of an interval $\omega_{p_{1}, \ldots, p_{s}} \in \widehat{\mathcal{P}}$ with a given combinatorics. In Section 3.3 we combine these with some counting arguments to obtain estimates on $\{\hat{p}>n \mid J\}$. Note that the supremum of $\{\hat{p}>n \mid J\}$, when taken over all intervals $J$, will be infinite, because tiny intervals take a long time to reach large scale. When applying Proposition 3.1 in Section 4, we will fix the minimal interval length $\delta^{\prime \prime}:=\min \left\{\delta^{\prime} / 3,\left|\Omega_{0}\right|\right\}$, where $\Omega_{0}$ is an interval specified in Section 4.1. In this way, we obtain a bound of

$$
\left\{|\{\hat{p}>n \mid J\}| ;|J| \geqslant \delta^{\prime \prime}\right\}
$$

which depends only on $\delta^{\prime \prime}$, that is: the $\widehat{C}$ 's in Proposition 3.1 depend on $\delta^{\prime \prime}$ but not on $J$.

\subsection{Combinatorial structure of $\widehat{F}$}

We start with any interval $\omega \in \mathcal{P} \mid J$, i.e. $\omega=I_{p} \cap J$ for some $p \geqslant 0$, and let

$$
\nu_{1}=\min \left\{n \geqslant 0 ; f^{n}(\omega) \cap \Delta \neq \emptyset\right\}
$$



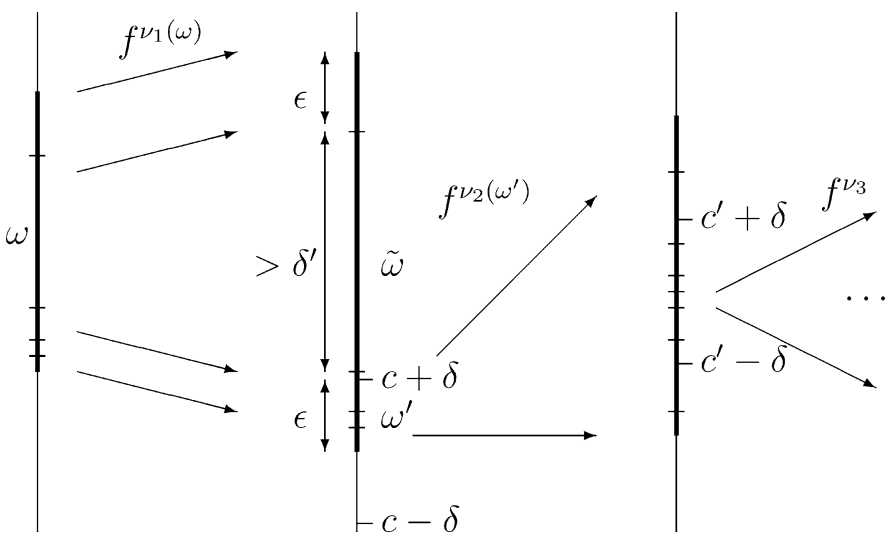

Fig. 1. Construction of partitions $\widehat{\mathcal{P}}_{n}$. The middle part of $\omega$ reaches large scale after $\nu_{1}$ iterates; the upper part has a shallow return, and the lower part has a deep return.

be the first visit to $\Delta$. Write $\tilde{\omega}=f^{\nu_{1}}(\omega)$. There are two (mutually exclusive) cases:

- $|\tilde{\omega}|<\delta^{\prime}$. We partition $\tilde{\omega}$ by intersecting $\tilde{\omega}$ with the elements $\left\{I_{p}\right\}$. Each interval $I_{p} \cap \tilde{\omega}$ for $p>0$ is labeled as Deep Return. The interval $I_{0} \cap \tilde{\omega}$ is taken together with the interval $I_{p} \cap \tilde{\omega}$ adjacent to it. Because $\tilde{\omega}$ is not too large compared to this particular component of $I_{p}$, the estimates of the binding period of $I_{p}$ go through, see Lemma 2.6 and the remark below it.

- $|\tilde{\omega}| \geqslant \delta^{\prime}$. We cut off the outmost intervals of length $\varepsilon$ from $\tilde{\omega}$, and stop with the remaining middle part; it has reached large scale. The subinterval $\omega_{0} \subset \omega$ such that $f^{\nu_{1}}\left(\omega_{0}\right)$ equals this middle part of $\tilde{\omega}$ is added to the partition $\widehat{\mathcal{P}}$. Here $\varepsilon \ll \delta^{\prime}$ is a constant to be fixed in the proof of Lemma 3.6. The $\varepsilon$ will be used effectively in Lemma 4.2. For the moment it suffices to know that $\varepsilon$ is smaller than each component of $\Delta$ and each component of $X \backslash \Delta$. The outmost intervals $\tilde{\omega}_{ \pm}$of length $\varepsilon$ are partitioned by intersecting them with the elements $\left\{I_{p}\right\}$. Each interval $I_{p} \cap \tilde{\omega}_{ \pm}$for $p>0$ is labeled as Deep Return. The interval $I_{0} \cap \tilde{\omega}_{ \pm}$(if it exists) is labeled as Shallow Return.

Note that if $x \in \omega$, then $f^{n}(x) \in \Delta$ only if $n$ is in a binding period of $x, x$ has a deep return or if $n=\hat{p}(x)$. At shallow return times $n, f^{n}(x) \notin \Delta$.

Now let $\omega^{\prime}$ be an interval which results from this partitioning of $\tilde{\omega}$, which has not reached large scale, see Fig. 1. We first apply the binding period, i.e. we take $f^{p}\left(\omega^{\prime}\right)$ for the stopping time $p=p\left(\omega^{\prime}\right)$ (which is possibly 0 , namely if $\omega^{\prime} \cap \Delta=\emptyset$ ), and then take the second return $\nu_{2}=\min \left\{n \geqslant p\left(\omega^{\prime}\right) ; f^{n}\left(\omega^{\prime}\right) \cap \Delta \neq \emptyset\right\}$. Subdivide $f^{\nu_{2}}\left(\omega^{\prime}\right)$ according to the above rules, distinguishing between large and deep returns.

Let $\widehat{\mathcal{P}}_{n}$ be the partition which we obtain by only considering at most $n$ iterates of $f$ and $\widehat{\mathcal{P}}$ the partition of $J$ by considering all iterates of $f$. We should emphasize that the procedure and hence the partitions depend on the choice made for $J$ and on $\varepsilon$ ). For example, if $J_{1}$ and $J_{2}$ are two intersecting intervals, then one could get two different partitions created at a point $x \in J_{1} \cap J_{2}$.

Next we define the stopping time at large scale $\hat{p}_{J}$ : At points $x \in \omega$ where the procedure eventually stops, i.e., for which there exists $n>0$ so that the $n$th iterate of the interval in $\widehat{\mathcal{P}}_{n}$ containing $x$ has reached large scale, set $\hat{p}_{J}(x)=n$. At other points $x \in J$ set $\hat{p}_{J}(x)=\infty$. Finally to define $\widehat{F}_{J}$, let $\hat{J}=\left\{x \in J: \hat{p}_{J}(x)<\infty\right\}$ and define $\widehat{F}_{J}: \hat{J} \rightarrow I$ by $\widehat{F}_{J}(x)=f^{\hat{p}_{J}(x)}(x)$. We shall prove that $\hat{J}=J$ up to sets of zero Lebesgue measure.

Take $n<\infty$. To each $\omega_{n} \in \widehat{\mathcal{P}}_{n}$ we assign a formal itinerary

$$
\left(\nu_{1}, p_{1}\right), \ldots,\left(\nu_{s}, p_{s}\right)
$$


consisting of the stopping times and lengths of the corresponding binding periods; $s$ is maximal for $\nu_{s} \leqslant n$. Depending on the depth of the return at time $\nu_{s}, \nu_{s}+p_{s}$ can be arbitrarily large. If the return at time $\nu_{j}$ is shallow, then $p_{j}=0$. If $\omega$ is an interval on which $\left(\nu_{1}, p_{1}\right), \ldots,\left(\nu_{j-1}, p_{j-1}\right)$ is constant and for which $\nu_{j}=\nu_{j}(\omega)$ is the next return to $\Delta$, then the set $\left\{x \in \omega ; p_{j}(x)=p\right\}$ has at most 4 components. This maximum is attained when $\left|f^{\nu_{j}(\omega)}\right| \geqslant \delta$, the radius of $\Delta$, and the outmost intervals of size $\varepsilon$ both contain a critical point. It can happen that $f^{\nu_{j}}(\omega)$ covers many more critical points, but since $\omega$ has reached large scale, $p_{j}$ is only defined on the outmost intervals. We will take care of this multiplicity in the estimates in Section 3.3. But apart from this multiplicity, a sequence $p_{1}, \ldots, p_{s}$ uniquely determines a partition element $\omega_{p_{1}, \ldots, p_{s}} \in \widehat{\mathcal{P}}_{n}$ (or perhaps the empty set). Indeed, $p_{i}$ determines the position of the $i$ th return of $\omega_{p_{1}, \ldots, p_{s}}$, and from the previous $p_{1}, \ldots, p_{i-1}$ and the starting interval $J$ one can compute the next return time. Hence the information $\nu_{1}, \ldots, \nu_{s}$ is strictly speaking superfluous. Observe however that there are many itineraries that do not correspond to partition elements. Note that $f^{n}$ is a diffeomorphism on each interval from the partition $\widehat{\mathcal{P}}_{n}$.

For a given sequence $\left(p_{1}, \ldots, p_{s}\right)$, let

$$
S_{d}=\left\{i \leqslant s ; \nu_{i} \text { is a deep return }\right\}=\left\{i \leqslant s ; p_{i}>0\right\}
$$

and

$$
S_{s}=\left\{i \leqslant s ; \nu_{i} \text { is a shallow return }\right\}=\left\{i \leqslant s ; p_{i}=0\right\}=S \backslash S_{d} .
$$

Moreover, let

$$
S_{s, s}=\left\{i<s ; p_{i}=0 \text { and } p_{i+1}=0\right\} .
$$

Because each index in $S_{s} \backslash S_{s, s}$ either equals $s$ or is followed by an index in $S_{d}$, we get

$$
\# S_{s} \leqslant \# S_{s, s}+\# S_{d}+1
$$

\subsection{Metric and combinatorial estimates}

LemmA 3.2. - Let $C=C_{\delta}$ and $\lambda=\lambda_{\delta}$ be as in Lemma 2.4. There exist $K_{0}>0$ independent of $\varepsilon$ and $\rho \in(0,1)(\rho \rightarrow 0$ as $\varepsilon \rightarrow 0)$, with the following properties. For a given sequence $\left(\nu_{1}, p_{1}\right), \ldots\left(\nu_{s}, p_{s}\right)$ with $\nu_{s} \leqslant n$ we have

$$
\frac{\left|\omega_{p_{1}, \ldots, p_{s}}\right|}{\left|f^{m}\left(\omega_{p_{1}, \ldots, p_{s}}\right)\right|} \leqslant \min \left\{C^{-\# S_{d}} \mathrm{e}^{-\lambda\left(m-\sum_{i=0}^{s} p_{i}\right)},\left(\frac{K_{0}}{\kappa}\right)^{\# S_{d}} \rho^{\# S_{s, s}}\right\} \prod_{i \in S_{d}}\left(F_{p_{i}}^{\prime}\right)^{-1}
$$

for $m=\max \left\{n, \nu_{s}+p_{s}\right\}$. Moreover there exists $T>0$ which can be chosen arbitrarily large if $\varepsilon$ is small, such that $\nu_{i+1}-\nu_{i} \geqslant T$ whenever $p_{i}=p_{i+1}=0$.

Proof. - By construction, $f^{m} \mid \omega_{p_{1}, \ldots, p_{s}}$ is a diffeomorphism onto its image. Take $x \in \omega_{p_{1}, \ldots, p_{s}}$ and divide its orbit into pieces separated by returns (both deep and shallow):

$$
\left[1, \nu_{1}-1\right],\left[\nu_{1}, \nu_{2}-1\right],\left[\nu_{2}, \nu_{3}-1\right], \ldots,\left[\nu_{s}, m\right] \text {. }
$$

Let $\nu_{i}<\nu_{i^{\prime}}$ be two consecutive deep returns. That is, assume that $p_{i}>0, p_{i+1}=\cdots=p_{i^{\prime}-1}=0$ and $p_{i^{\prime}}>0$ (with possibly $i^{\prime}=i+1$ ). Because each such interval lasts at least the corresponding binding period, and in the remaining time the point $x$ does not visit $\Delta$, Lemma 2.4 and the 
definition of $F_{p}^{\prime}$ give

$$
\left|\left(f^{\nu_{i^{\prime}}-\nu_{i}}\right)^{\prime}\left(f^{\nu_{i}}(x)\right)\right| \geqslant C \mathrm{e}^{\lambda\left(\nu_{i^{\prime}}-\left(\nu_{i}+p_{i}\right)\right)} F_{p_{i}}^{\prime}
$$

Hence the chain rule and the Mean Value Theorem show that

$$
\left|\omega_{p_{1}, \ldots, p_{s}}\right| \leqslant C^{-\# S_{d}} \mathrm{e}^{-\lambda\left(m-\sum p_{i}\right)}\left|f^{m}\left(\omega_{p_{1}, \ldots, p_{s}}\right)\right| \prod_{i} 1 / F_{p_{i}}^{\prime} .
$$

To prove the other inequality, let $\nu_{i}$ and $\nu_{i^{\prime}}$ be subsequent deep return times. First let us treat the step from $\nu_{i^{\prime}-1}+p_{i^{\prime}-1}$ to $\nu_{i^{\prime}}$, so assume that $\nu_{i^{\prime}-1}+p_{i^{\prime}-1}<\nu_{i^{\prime}}$. (If $\nu_{i^{\prime}-1}+p_{i^{\prime}-1}=\nu_{i^{\prime}}$ we can skip this step.) Therefore $f^{\nu_{i^{\prime}-1}+p_{i^{\prime}-1}}\left(\omega_{p_{1}, \ldots, p_{s}}\right) \cap \Delta=\emptyset$, while there is at least one point $y \in \omega_{p_{1}, \ldots, p_{s}}$ such that $f^{\nu^{\prime}}(y) \in \Delta$. Lemma 2.3 yields that

$$
\left|\left(f^{\nu_{i^{\prime}}-\left(\nu_{i^{\prime}-1}+p_{i^{\prime}-1}\right)}\right)^{\prime}\left(f^{\nu_{i^{\prime}-1}+p_{i^{\prime}-1}}(y)\right)\right| \geqslant \kappa .
$$

Because $\nu_{i^{\prime}}<n,\left|f^{\nu_{i^{\prime}}}\left(\omega_{p_{1}, \ldots, p_{s}}\right)\right|<\delta^{\prime}$. Take $H \supset f^{\nu_{i^{\prime}-1}+p_{i^{\prime}-1}}\left(\omega_{p_{1}, \ldots, p_{s}}\right)$ the largest interval on which $f^{\nu_{i^{\prime}}-\left(\nu_{i^{\prime}-1}+p_{i^{\prime}-1}\right)}$ is monotone. Then by the choice of $\delta^{\prime}, f^{\nu_{i^{\prime}}-\left(\nu_{i^{\prime}-1}+p_{i^{\prime}-1}\right)}(H)$ contains a $\frac{1}{3}$-scaled neighbourhood of $f^{\nu_{i^{\prime}}}\left(\omega_{p_{1}, \ldots, p_{s}}\right)$. Therefore the derivative of $f^{\nu_{i^{\prime}}-\left(\nu_{i^{\prime}-1}+p_{i^{\prime}-1}\right)}$ has distortion bounded by some $K_{0}=K_{0}\left(\frac{1}{3}\right) \leqslant 16$. This follows from the Koebe Lemma, see [19, Chapter IV]. Hence

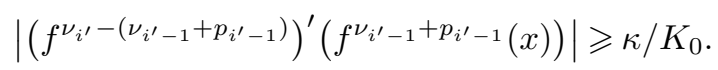

If $i^{\prime} \leqslant i+2$, the same argument gives $\left|\left(f^{\nu_{i^{\prime}}-\left(\nu_{i}+p_{i}\right)}\right)^{\prime}\left(f^{\nu_{i^{\prime}-1}+p_{i^{\prime}-1}}(x)\right)\right| \geqslant \kappa / K_{0}$, and indeed in this case there are no entries of $S_{s, s}$ between $i$ and $i^{\prime}$.

If $i^{\prime}>i+2$, then the differences $\nu_{i+2}-\left(\nu_{i}+p_{i}\right), \nu_{i+3}-\nu_{i+2}, \ldots, \nu_{i^{\prime}-1}-\nu_{i^{\prime}-2}$ are all large if $\varepsilon$ is small. Indeed, in these times an interval of size $\varepsilon$ must have expanded to an interval of size $\delta^{\prime} \gg \varepsilon$. Because $x$ does not visit $\Delta$ during these iterates (recall that the binding periods at shallow returns have length 0 ), the first part of Lemma 2.4 gives

$$
\left|\left(f^{\nu_{i^{\prime}-1}-\left(\nu_{i}+p_{i}\right)}\right)^{\prime}\left(f^{\nu_{i}+p_{i}}(x)\right)\right| \geqslant\left(\frac{1}{\rho}\right)^{i^{\prime}-(i+2)},
$$

where $\rho \rightarrow 0$ as $\varepsilon \rightarrow 0$. (In Lemma 3.6 we will fix $\rho$ at $1 / 8$.) Adding the numbers $i^{\prime}-i-2$ (running over all pairs $\left(i^{\prime}, i\right)$ of subsequent deep returns with $i^{\prime}>i+2$ ) gives $\# S_{s, s}$. This proves the lemma.

LEMMA 3.3. - There exists $K>0$ depending only on $\varepsilon$ such that for all starting intervals $J$ and $\omega \in \widehat{\mathcal{P}}$, the distortion of $\widehat{F}_{J} \mid \omega$ is bounded by $K$.

Proof. - If $\hat{p}(\omega)=n$, then by construction, there is an interval $T \supset \omega$ such that $f^{n}$ maps $T$ monotonically onto an $\varepsilon$-scaled neighbourhood of $f^{n}(\omega)$, i.e. both components of $f^{n}(T) \backslash f^{n}(\omega)$ have size $\geqslant \varepsilon\left|f^{n}(\omega)\right|$. The Koebe Principle (see e.g. [19] and in the setting when we do not assume $S f<0$, [24]) gives the result.

The following lemma contains combinatorial estimates needed in the next section.

LEMMA 3.4. - Let $N_{k, s}$ be the number of integer sequences $\left(p_{1}, \ldots, p_{s}\right)$ such that

$$
p_{1}+\cdots+p_{s}=k \text { and } p_{i} \geqslant 0 \text { for all } i
$$


Let $N_{k, s}^{+}$be the same number for sequences with $p_{i}>0$ for all $i$. Then

$$
N_{k, s} \leqslant 2^{s} \max _{j \leqslant k} N_{k, j}^{+}<2^{k+s-1} .
$$

Given $\zeta>0$ small and $\alpha \in(0,1]$, there exists $\hat{\zeta}=\hat{\zeta}(\zeta, \alpha)$ with $\hat{\zeta} \rightarrow 0$ as $\zeta \rightarrow 0$ such that

$$
N_{k, s}^{+} \leqslant \begin{cases}\mathrm{e}^{\hat{\zeta} k^{\alpha} \log k} & \text { if } s \leqslant \zeta k^{\alpha} \\ \mathrm{e}^{\hat{\zeta} k} & \text { if } s \leqslant \zeta k\end{cases}
$$

Proof. - Say $j \geqslant 1$ terms in the sum $p_{1}+\cdots+p_{s}$ are nonzero. There are $\left(\begin{array}{l}s \\ j\end{array}\right)$ ways to distribute them. This gives

$$
N_{k, s}=\sum_{j=1}^{s}\left(\begin{array}{l}
s \\
j
\end{array}\right) N_{k, j}^{+} \leqslant \max _{j} N_{k, j}^{+} \sum_{j=1}^{s}\left(\begin{array}{l}
s \\
j
\end{array}\right)=\left(2^{s}-1\right) \max _{j} N_{k, j}^{+} .
$$

Next, supposing that $p_{i}>0$ for all $i$, there are $s-1$ partial sums $\sum_{i=1}^{j} p_{i}$ different from $k$. Therefore $N_{k, j}^{+}=\left(\begin{array}{c}k-1 \\ j-1\end{array}\right)$. In particular, $\max _{j} N_{k, j}^{+} \leqslant 2^{k-1}$. This proves (10). Let us estimate $N_{k, s}^{+}$more precisely if $s \leqslant \zeta k^{\alpha} \leqslant k / 2$. By Stirling's formula

$$
\left(\begin{array}{l}
k \\
s
\end{array}\right) \leqslant \frac{k^{k}}{\left(s^{s}\right)(k-s)^{k-s}} \leqslant\left(\frac{k}{k-s}\right)^{k} \cdot\left(\frac{k-s}{s}\right)^{s} \leqslant\left(1+\frac{2 s}{k}\right)^{k} \cdot\left(\frac{k}{s}\right)^{s} .
$$

Because $\left(1+\frac{2 s}{k}\right)^{k} \leqslant \exp \left(k \log \left(1+\frac{2 s}{k}\right)\right) \leqslant \exp (2 s)$, it follows that

$$
N_{k, s}^{+}<\mathrm{e}^{2 s}\left(\frac{k^{1-\alpha}}{\zeta}\right)^{\zeta k^{\alpha}} \leqslant \mathrm{e}^{\zeta(2-\log \zeta+(1-\alpha) \log k) k^{\alpha}}
$$

if $s \leqslant \zeta k^{\alpha}$. If $s \leqslant \zeta k$, this simplifies to $N_{k, s}^{+}<\mathrm{e}^{\zeta(2-\log \zeta) k}$, proving (11).

\subsection{Stopping time estimates}

The aim of this section is to estimate the tail behaviour of the return time function $\hat{p}$, i.e. to obtain an upper bound for the Lebesgue measure of the set $\{x \in J: \hat{p}(x)>n\}$ which we shall henceforth (suppressing the dependence on $J$ ) denote by $\{\hat{p}>n\}$. We shall always assume the notation of Proposition 3.1, particularly when referring to the polynomial, stretched exponential and exponential cases.

We fix $n$ for the rest of this section. For each $\omega \in \widehat{\mathcal{P}}$ we consider the sequence $p_{1}, \ldots, p_{s}$ as defined above, with some terms possibly equal to 0 . Recall that $s$ is given by the number of returns occurring before time $n$. Let $\eta>0$ be a small constant to be determined in Lemma 3.5. The set of partition elements $\omega \in \widehat{\mathcal{P}}_{n}$ with $\hat{p} \mid \omega>n$ can be divided into

$$
\widehat{\mathcal{P}}_{n}^{\prime}=\left\{\omega \in \widehat{\mathcal{P}}_{n} ; \hat{p} \mid \omega>n, \sum_{i=1}^{s} p_{i} \leqslant \eta n\right\}
$$

and

$$
\widehat{\mathcal{P}}_{n}^{\prime \prime}=\left\{\omega \in \widehat{\mathcal{P}}_{n} ; \hat{p} \mid \omega>n, \sum_{i=1}^{s} p_{i}>\eta n\right\}
$$


Clearly we have

$$
|\{\hat{p}>n\}|=\sum_{\omega \in \widehat{\mathcal{P}}_{n}^{\prime}}|\omega|+\sum_{\omega \in \widehat{\mathcal{P}}_{n}^{\prime \prime}}|\omega| .
$$

To treat the exponential and stretched exponential cases we shall need to subdivide $\widehat{\mathcal{P}}_{n}^{\prime \prime}$ further into

$$
\widehat{\mathcal{P}}_{n-}^{\prime \prime}=\left\{\omega \in \widehat{\mathcal{P}}_{n}^{\prime \prime} ; s \leqslant \rho n^{\hat{\alpha}}\right\} \quad \text { and } \quad \widehat{\mathcal{P}}_{n+}^{\prime \prime}=\left\{\omega \in \widehat{\mathcal{P}}_{n}^{\prime \prime} ; s>\rho n^{\hat{\alpha}}\right\},
$$

where $\hat{\alpha} \in(0,1]$ and $\rho>0$ will be chosen below.

Intuitively elements in $\widehat{\mathcal{P}}_{n}^{\prime}$ spend most of their time in the "uniformly expanding" region $X \backslash \Delta$. Thus intervals are growing in size at a uniform exponential rate and achieve large scale exponentially fast. Elements of $\widehat{\mathcal{P}}_{n}^{\prime \prime}$ on the other hand spend much time in binding periods. In this case the upper bound will more closely reflect the expanding properties of the critical orbit. We shall apply various combinations of the estimates obtained in Section 3.2 to obtain bounds on the total measure of the elements of the subpartitions defined above under the required assumptions on the growth of $D_{n}$.

LEMMA 3.5. - For any $\theta>0$ there exists $\eta_{0}>0$ such that for all $0<\eta<\eta_{0}$ and for all $n$ sufficiently large,

$$
\sum_{\omega \in \widehat{\mathcal{P}}_{n}^{\prime}}|\omega| \leqslant \mathrm{e}^{-(\lambda-\theta) n} .
$$

Proof. - As in Lemma 3.4, let $N_{k, s}$ denote the number of possible sequences $\left(p_{1}, \ldots, p_{s}\right)$ with $p_{i} \geqslant 0$ and $p_{1}+\cdots+p_{s}=k$. Then by the definition of $\widehat{\mathcal{P}}^{\prime}$ and the first statement of Lemma 3.2 we have

$$
\sum_{\omega \in \widehat{\mathcal{P}}^{\prime}}|\omega| \leqslant \sum_{s=1}^{n} \sum_{k=0}^{\eta n} \sum_{\substack{\left.p_{1}, \ldots, p_{s}\right) \\ \sum p_{i}=k}}\left|\omega_{p_{1}, \ldots, p_{s}}\right| \leqslant \sum_{s=1}^{n} \sum_{k=0}^{\eta n} 4^{s} N_{k, s} C^{-s} \mathrm{e}^{-\lambda(1-\eta) n} .
$$

Here the factor $4^{s}$ expresses the maximal number of components of $I_{p}$ for each return, see the argument in Section 3.1. We use the bound $N_{k, s}<2^{k+s}$ from Lemma 3.4. Recall that $k \leqslant \eta n$. Since $\nu_{i+1}-\nu_{i} \geqslant T$ when $p_{i}=p_{i+1}=0$ (see the previous lemma) formula (9) gives $s=\# S_{d}+\# S_{s} \leqslant 2 \# S_{d}+\# S_{s, s}+1 \leqslant 2 \eta n+n / T+1$. So in (12), $s$ only ranges up to this bound. Writing $\eta^{\prime}=(3 \eta+1 / T+1 / n)\left(3 \log 2+\log C^{-1}\right)$, we get $4^{s} N_{k, s} C^{-s} \leqslant \mathrm{e}^{\eta^{\prime} n}$. Taking $\theta=2\left(\eta+\eta^{\prime} / \lambda\right)$ and substituting in (12) gives

$$
\sum_{\omega \in \widehat{\mathcal{P}}_{n}^{\prime}}|\omega| \leqslant n \sum_{k=1}^{\eta n} \mathrm{e}^{\eta^{\prime} n} \mathrm{e}^{-\lambda(1-\eta) n} \leqslant \eta n^{2} \mathrm{e}^{-\left(\lambda-\frac{\theta}{2}\right) n} \leqslant \mathrm{e}^{-(\lambda-\theta) n}
$$

provided $\eta$ and $\eta^{\prime}$ are sufficiently small and $n$ sufficiently large.

Lemma 3.6. - Recall from (2) that $d_{n}(c)=\min _{i<n}\left(\gamma_{i} / D_{i}(c)\right)^{1 / \ell}\left|f^{i}(c)-\mathcal{C}\right|$. Fix $L \in$ $\{1, \ldots, n\}$ arbitrary and let

$$
\hat{d}_{n, s}(c)=d_{i}(c) \quad \text { for } i=\max \left\{\left\lceil\frac{\eta n}{2 s^{2}}\right\rceil, L\right\}
$$


Write $s(\omega)=s$ if the itinerary $\left(p_{1}, \ldots, p_{s}\right)$ of $\omega$ has length $s$. For any $\eta>0$ there exists $C_{1}>0$ such that

$$
\sum_{\substack{\omega \in \widehat{\mathcal{P}}_{n}^{\prime \prime} \\ s(\omega) \geqslant L}}|\omega| \leqslant C_{1} \max _{c \in \mathcal{C}} \sum_{s=L}^{n} 2^{-s} \hat{d}_{n, s}(c) .
$$

Proof. - Given a sequence $\left(p_{1}, \ldots, p_{s}\right)$, let $p_{j^{\prime}}$ be the first term such that $p_{j^{\prime}} \geqslant \eta n /\left(2 j^{\prime 2}\right)$. Because $p_{1}+\cdots+p_{s} \geqslant \eta n$, such $j^{\prime}$ exists. Take $j=\max \left\{L, j^{\prime}\right\}$.

Let $\tilde{\omega}_{p_{1}, \ldots, p_{j}}$ be the union of adjacent intervals $\omega_{p_{1}, \ldots, p_{j-1}, p}$ with common return times $\nu_{1}, \ldots, \nu_{j}$ and $p \geqslant p_{j}$. Then $f^{\nu_{j}}$ maps $\tilde{\omega}_{p_{1}, \ldots, p_{j}}$ diffeomorphically into an interval $(x, y)$ such that $p(x), p(y) \geqslant p_{j}$. Assume without loss of generality that $|x-c| \geqslant|y-c|$. Therefore, for each $i<p_{j}$,

$$
\begin{aligned}
\gamma_{i}\left|f^{i}(c)-\mathcal{C}\right| & \geqslant\left|f^{i}(x)-f^{i}(c)\right| \geqslant \Gamma^{-1} D_{i-1}(c)|f(x)-f(c)| \\
& \geqslant \mathcal{O}(1 / \Gamma) D_{i-1}(c)|x-c|^{\ell} \geqslant \mathcal{O}(1 / \Gamma) \frac{D_{i}(c)|x-c|^{\ell}}{\left|f^{i}(c)-\mathcal{C}\right|^{\ell-1}}
\end{aligned}
$$

This gives

$$
|x-y| \leqslant 2|x-c| \leqslant \mathcal{O}\left(2 \Gamma^{1 / \ell}\right) d_{p}(c) \leqslant \mathcal{O}\left(2 \Gamma^{1 / \ell}\right) \max _{p \geqslant \eta n / 2 j^{2}} d_{p}(c)=\mathcal{O}\left(2 \Gamma^{1 / \ell}\right) \hat{d}_{n, j}(c) .
$$

Let $S_{s}$ and $S_{d}$ be the indices $\leqslant j$ corresponding to shallow respectively deep returns. Also let $S_{d}^{\prime}=S_{d} \backslash\{j\}$ and let $S_{s, s}$ be the indices $\leqslant j$ of shallow returns that are followed by another shallow return. Now Lemma 3.2 applied to $\tilde{\omega}_{p_{1}, \ldots, p_{j}}$ and the iterate $\nu_{j}$ gives

$$
\begin{aligned}
\sum_{\substack{\omega \in \widehat{\mathcal{P}}_{n}^{\prime \prime} \\
s(\omega) \geqslant L}}|\omega| & \leqslant \sum_{j=L}^{n} \sum_{\left(p_{1}, \ldots, p_{j}\right)}\left|\tilde{\omega}_{p_{1}, \ldots, p_{j}}\right| \\
& \leqslant \sum_{j=L}^{n} \mathcal{O}\left(2 \Gamma^{1 / \ell}\right) \max _{c \in \mathcal{C}} \hat{d}_{n, j}(c) \sum_{\left(p_{1}, \ldots, p_{j-1}\right)} 4^{j}\left(\frac{K_{0}}{\kappa}\right)^{\# S_{d}} \rho^{\# S_{s, s}} \prod_{i \in S_{d}^{\prime}} \frac{1}{F_{p_{i}}^{\prime}} .
\end{aligned}
$$

The factor $4^{j}$ expresses the different components of the level sets $I_{p}$ that intersect forward iterates of $\omega$ (see the argument in Section 3.1), and the factor $\left(K_{0} / \kappa\right)^{\# S_{d}} \rho^{\# S_{s, s}}$ comes from Lemma 3.2. Using (9) and the fact that $\# S_{d}^{\prime}=\# S_{d}-1$ we can write $4^{j}=2^{-j} 8^{j}$ and

$$
8^{j}=8^{\# S_{s}+\# S_{d}} \leqslant 8^{\# S_{s, s}+2 \# S_{d}+1}=8^{\# S_{s, s}} 64^{\# S_{d}} 8=5128^{\# S_{s, s}} 64^{\# S_{d}^{\prime}} .
$$

Then

$$
\sum_{\left(p_{1}, \ldots, p_{j-1}\right)} 4^{j}\left(\frac{K_{0}}{\kappa}\right)^{\# S_{d}} \rho^{\# S_{s, s}} \prod_{i \in S_{d}^{\prime}} \frac{1}{F_{p_{i}}^{\prime}} \leqslant 2^{-j} \frac{512 K_{0}}{\kappa} \sum_{\left(p_{1}, \ldots, p_{j-1}\right)}(8 \rho)^{\# S_{s, s}} \prod_{i \in S_{d}^{\prime}} \frac{64 K_{0}}{\kappa F_{p_{i}}^{\prime}} .
$$

Take $\varepsilon$ in Lemma 3.2 so small that $\rho=\frac{1}{8}$ and recall that $p_{i} \geqslant p_{\delta}$ for all $i \in S_{d}$. Therefore Lemma 2.5 and formula (5) (with $\zeta=64 K_{0} / \kappa$ ) give that

$$
\sum_{\left(p_{1}, \ldots, p_{j-1}\right)}(8 \rho)^{\# S_{s, s}} \prod_{i \in S_{d}^{\prime}} \frac{64 K_{0}}{\kappa F_{p_{i}}^{\prime}} \leqslant 1
$$


By (6), the lemma follows with $C_{1}=\mathcal{O}\left(2 \Gamma^{1 / \ell}\right) 512 K_{0} / \kappa$.

The previous lemma is not so useful in the exponential and stretched exponential cases for relatively small values of $s$. Indeed, consider for example the situation that $d_{p}=\mathrm{e}^{-\beta p}$. Then the term in the sum in Lemma 3.6 corresponding to $s=\sqrt{n}$ gives $C_{1} 2^{-\sqrt{n}} \cdot \mathrm{e}^{-\eta \beta / 2}$. Clearly this decreases merely subexponentially in $n$. Let us improve on this.

LEMma 3.7. - Assume that there exist $C, \beta>0$ and $\alpha \in(0,1]$ such that $D_{n}^{-1 / \ell} \leqslant C \mathrm{e}^{-\beta n^{\alpha}}$ for all $n$. Then for each $\hat{\alpha} \in(0, \alpha)$ (or $\hat{\alpha}=1$ if $\alpha=1$ ) there exist $\rho, C^{\prime}, \beta^{\prime}>0$ such that

$$
\sum_{\omega \in \widehat{\mathcal{P}}_{n-}^{\prime \prime}}|\omega| \leqslant C^{\prime} \mathrm{e}^{-\beta^{\prime} n^{\alpha}}
$$

for all $n$. Note that the set $\mathcal{P}_{n-}^{\prime \prime}$ depends on $\rho$ and $\hat{\alpha}$.

Proof. - First notice that since $\alpha \in(0,1]$ one has $p_{1}^{\alpha}+p_{2}^{\alpha} \geqslant\left(p_{1}+p_{2}\right)^{\alpha}$. Using Lemmas 2.5 and 3.2 this gives that there exist $\beta^{\prime \prime}>0$ and $C$ such that

$$
\left|\omega_{p_{1}, \ldots, p_{s}}\right| \leqslant C^{-s} \prod_{i=1}^{s} \frac{1}{F_{p_{i}}^{\prime}} \leqslant C^{-s} C_{0}^{-s} \prod_{i=1}^{s} \max _{c \in \mathcal{C}} b_{p_{i}}(c) \leqslant C^{-s} \mathrm{e}^{-\beta^{\prime \prime}\left(\sum p_{i}\right)^{\alpha}} .
$$

Reasoning as in the proof of Lemma 3.5, we write $k=p_{1}+\cdots+p_{s}$ and we obtain

$$
\sum_{\omega \in \widehat{\mathcal{P}}_{n-}^{\prime \prime}}|\omega| \leqslant \sum_{s=1}^{\rho n^{\hat{\alpha}}} \sum_{k=\eta n}^{\infty} 4^{s} N_{k, s} C^{-s} \mathrm{e}^{-\beta^{\prime \prime} k^{\alpha}}
$$

Taking $\zeta=\rho / \eta^{\hat{\alpha}}$ respectively $\zeta=\rho / \eta$ in Lemma 3.4, we get that for some $\hat{\rho}=\hat{\rho}(\rho, \eta, \hat{\alpha})$ with $\hat{\rho} \rightarrow 0$ as $\rho \rightarrow 0$,

$$
N_{k, s} \leqslant \begin{cases}2^{s} \mathrm{e}^{\hat{\rho} k^{\hat{\alpha}} \log k} & \text { if } s \leqslant\left(\rho / \eta^{\hat{\alpha}}\right) k^{\hat{\alpha}}, \\ 2^{s} \mathrm{e}^{\hat{\rho} k} & \text { if } s \leqslant(\rho / \eta) k .\end{cases}
$$

(The second case applies when $\alpha=1$.) Because $\hat{\alpha} \leqslant \alpha$ and taking $\rho$ and therefore $\hat{\rho}$ sufficiently small, we get in either case

$$
\sum_{k=\eta n}^{\infty} 4^{s} N_{k, s} C^{-s} \mathrm{e}^{-\beta^{\prime \prime} k^{\alpha}} \leqslant 8^{s} C^{-s} \mathrm{e}^{-\beta^{\prime \prime}(\eta n)^{\alpha} / 2}
$$

Using again that $\hat{\alpha} \leqslant \alpha$ and the fact that $\rho$ is small, inequality (13) gives

$$
\sum_{\omega \in \widehat{\mathcal{P}}_{n-}^{\prime \prime}}|\omega| \leqslant \sum_{s=1}^{\rho n^{\hat{\alpha}}} \sum_{k=\eta n}^{\infty} 4^{s} N_{k, s} C^{-s} \mathrm{e}^{-\beta^{\prime \prime} k^{\alpha}} \leqslant C^{\prime} \mathrm{e}^{-\beta^{\prime \prime}(\eta n)^{\alpha} / 4}
$$

for some constant $C^{\prime}$. This proves the lemma with $\beta^{\prime}=\eta^{\alpha} \beta^{\prime \prime} / 4$.

Proof of Proposition 3.1. - We show first of all that $\hat{J}$ has full measure in $J$, i.e. $|\{\hat{p}>n\}| \rightarrow 0$ as $n \rightarrow \infty$. By (for example) Lemma 2.4, it follows that almost all $x \in J, f^{n}(x)$ accumulates 
onto $\mathcal{C}$. Hence $x$ has infinitely many deep return times, and it is contained in sets of the form $\omega_{p_{1}, \ldots, p_{s}}$ for itineraries of arbitrary length $s$. Because $\hat{p}\left(\omega_{p_{1}, \ldots, p_{s}}\right) \geqslant s \rightarrow \infty$ as $s \rightarrow \infty$, the proofs of Lemmas 3.5 and 3.6 show that $\sum_{\left(p_{1}, \ldots, p_{s}\right)}\left|\omega_{p_{1}, \ldots, p_{s}}\right| \rightarrow 0$ as $s \rightarrow \infty$. Therefore $|J \backslash \hat{J}|=0$.

To prove the remaining estimates in the four cases mentioned in the proposition, notice that we have exponential bounds for $\widehat{\mathcal{P}}_{n}^{\prime}$ and therefore we only need to concentrate here on $\widehat{\mathcal{P}}_{n}^{\prime \prime}$. The sequence $\left\{\hat{d}_{n, s}(c)\right\}$ is decreasing in $n$, and for each $k$ there are at most

$$
\#\left\{n ; k-1 \leqslant \eta n /\left(2 s^{2}\right) \leqslant k\right\} \leqslant 2 s^{2} / \eta
$$

numbers $n$ such that $k=\left[\eta n /\left(2 s^{2}\right)\right]$. Therefore, using Lemma 3.6 with $L=1$ :

$$
\begin{aligned}
\sum_{n \geqslant 1} \sum_{s=1}^{n} 2^{-s} \hat{d}_{n, s}(c) & \leqslant \sum_{s \geqslant 1} \frac{2 s^{2}}{\eta} 2^{-s} \sum_{k \geqslant 1}\left[\gamma_{k} / D_{k}(c)\right]^{1 / \ell}\left|f^{k}(c)-\mathcal{C}\right| \\
& \leqslant \frac{12}{\eta} \sum_{k \geqslant 1}\left[\gamma_{k} / D_{k}(c)\right]^{1 / \ell} \leqslant \frac{12}{\eta} \sum_{k \geqslant 1}\left[\gamma_{k}^{\ell-1} D_{k}(c)\right]^{-1 / \ell} .
\end{aligned}
$$

Hence the summable case follows from $(* *)$. Lemma 3.6 with $L=1$ gives for the polynomial case

$$
\sum_{\omega \in \mathcal{P}_{n}^{\prime \prime}}|\omega| \leqslant C_{1} \max _{c \in \mathcal{C}} \sum_{s=1}^{n} 2^{-s} \hat{d}_{n, s}(c) \leqslant C_{1} \sum_{s=1}^{n} 2^{-s}\left(\frac{2 s^{2}}{\eta n}\right)^{\alpha} \leqslant 12 C_{1} \eta^{-\alpha} n^{-\alpha}
$$

as required. In the exponential and stretched exponential cases we use Lemma 3.6 applied to $\widehat{\mathcal{P}}_{n+}^{\prime \prime}$ with $L=\rho n^{\hat{\alpha}}$ to get

$$
\sum_{\omega \in \mathcal{P}_{n+}^{\prime \prime}}|\omega| \leqslant C_{1} \max _{c \in \mathcal{C}} \sum_{s \geqslant \rho n^{\hat{\alpha}}} 2^{-s} \hat{d}_{n, s}(c) \leqslant C_{2} \mathrm{e}^{-(\log 2) \rho n^{\hat{\alpha}}}
$$

for some $C_{2}>0$. Lemma 3.7 takes care of the remaining collection $\mathcal{P}_{n-}^{\prime \prime}$.

\section{The full return map}

In this section we construct the full return map $\hat{f}: \Omega_{0} \rightarrow \Omega_{0}$ and carry out its tail estimates.

Proposition 4.1. - Suppose that $f$ satisfies $(*)$. Then for any $c \in \mathcal{C} \cap X$ there exist a neighbourhood $\Omega_{0}$ of $c$, a countable partition $\mathcal{Q}$ of $\Omega_{0}(\bmod 0)$ and a return time function $R: \mathcal{Q} \rightarrow \mathbb{N}$ with the following properties. For each $\omega \in \mathcal{Q}, \hat{f}:=f^{R}$ maps $\omega$ to $\Omega_{0}$ diffeomorphically with bounded distortion: letting

$$
s(x, y)=\min \left\{n ; \hat{f}^{n}(x), \hat{f}^{n}(y) \text { belong to different elements of } \mathcal{Q}\right\},
$$

there exist $\beta \in(0,1)$ and $C>0$ such that for all $\omega \in \mathcal{Q}$ and all $x, y \in \omega$,

$$
\left|\frac{\hat{f}^{\prime}(x)}{\hat{f}^{\prime}(y)}-1\right| \leqslant C \beta^{s(x, y)} .
$$

Moreover the tail $|\{R>n\}|$ of the return times satisfies the following estimates: 
Summable case: Under no conditions on $d_{n}(c)$ other than which stem from $(*)$

$$
\sum_{n}|\{R>n\}|<\infty
$$

Polynomial case: If $d_{n}(c) \leqslant C n^{-\alpha}$ for all $c \in \mathcal{C}$ and $n \geqslant 1$, then there exists $\widetilde{C}>0$ such that

$$
|\{R>n\}| \leqslant \widetilde{C} n^{-\alpha}
$$

Stretched exponential case: If $b_{n}(c) \leqslant C \mathrm{e}^{-\beta n^{\alpha}}, \alpha \in(0,1), \beta>0$ for all $c \in \mathcal{C}$ and $n \geqslant 1$, then for each $\tilde{\alpha} \in(0, \alpha)$ there exist $\tilde{\beta}, \widetilde{C}>0$ such that

$$
|\{R>n\}| \leqslant \widetilde{C} \mathrm{e}^{-\tilde{\beta} n^{\tilde{\alpha}}} .
$$

Exponential case: If $b_{n}(c) \leqslant C \mathrm{e}^{-\beta n}, \beta>0$ for all $c \in \mathcal{C}$ and $n \geqslant 1$, then there exist $\tilde{\beta}, \widetilde{C}>0$ such that

$$
|\{R>n\}| \leqslant \widetilde{C} \mathrm{e}^{-\tilde{\beta} n} .
$$

In Section 4.1 we explain how to choose $\Omega_{0}$ and how to define the partition $\mathcal{Q}$ and the return time function $R$. Notice that $R$ is not a first return time. In Section 4.2 we prove the distortion bound and in Section 4.3 we prove the estimates on the return times.

\subsection{Large scales and full returns}

Let $\Omega_{0} \subset \Delta$ be a small neighbourhood of a point $c \in \mathcal{C}$ (the precise requirements on its size will be given in the proof of Lemma 4.2 below). Let $J \subset X$ be an arbitrary interval. Consider the map $F=f^{\hat{p}}: J \rightarrow X$ and the associated partition $\widehat{\mathcal{P}}$ on $J$ with the stopping time function $\hat{p}$ as defined in Section 3.

LEMmA 4.2. - There exist $t_{0} \in \mathbb{N}$ and $\xi>0$ independent of $J$ such that for every $\omega \in \widehat{\mathcal{P}}$ there exists $\tilde{\omega} \subset \omega$ satisfying the following properties:

- $f^{\hat{p}(\omega)+t}$ maps $\tilde{\omega}$ diffeomorphically onto $\Omega_{0}$ for some $t \leqslant t_{0}$;

- $|\tilde{\omega}| \geqslant \xi|\omega|$

- both components of $f^{\hat{p}(x)}(\omega \backslash \tilde{\omega})$ have length $\geqslant \delta^{\prime} / 3$.

Proof. - By definition of $X$, the preimages of $c$ are dense in $X$. Therefore there exists $t_{0} \geqslant 1$ such that every interval of length $\geqslant \delta^{\prime}$ contains a point $x \in \bigcup_{t \leqslant t_{0}} f^{-t}(c)$ in its middle fifth. Say $f^{t}(x)=c$. Now choose sufficiently small neighbourhoods $\omega_{x}$ of each such $x$ not containing any points of $f^{-j}(\mathcal{C})$ for any $j<t$. Clearly $f^{t}$ maps $\omega_{x}$ diffeomorphically to some critical neighbourhood. By adjusting the size of $\omega_{x}$ we can make sure that they all (i.e. for all points $x$ ) map onto exactly the same critical neighbourhood $\Omega_{0}$ and that $\left|\omega_{x}\right| \leqslant \delta^{\prime} / 15$. Let $\tilde{\omega} \subset \omega$ be the interval that is mapped onto $\omega_{x}$ by $f^{\hat{p}(\omega)}$. This proves the first and third statement.

From Lemma 3.3 we know that the distortion $f^{\hat{p}(\omega)} \mid \omega$ is bounded by $K=K(\varepsilon)$. The second statement follows immediately.

Having fixed $\Omega_{0}$, let $\delta^{\prime \prime}=\min \left\{\delta^{\prime} / 3,\left|\Omega_{0}\right|\right\}$. In the remainder we will only need to consider intervals $J$ of size $\geqslant \delta^{\prime \prime}$.

We now define $\hat{f}: \Omega_{0} \rightarrow \Omega_{0}$, the associated partition $\mathcal{Q}$ and the stopping time function $R$ constant on elements of $\mathcal{Q}$ such that $\widehat{f}=f^{R(\omega)}$ on $\omega \in \mathcal{Q}$. For each $\omega$ in the partition $\widehat{\mathcal{P}}$ of $\Omega_{0}$, let $\tilde{\omega}$ denote the subinterval given in Lemma 4.2 , so $\left|f^{\hat{p}(\omega)}(\tilde{\omega})\right| \geqslant \delta^{\prime}$. We put $\tilde{\omega} \in \mathcal{Q}$ by 
definition and $R(\tilde{\omega})=\hat{p}(\omega)+t$. Both components of $f^{\hat{p}(\omega)}(\omega) \backslash \omega_{x}$ have size at least $\delta^{\prime} / 3$. Considering them as new starting intervals we carry out the construction of Section 3 and repeat the procedure described above. This determines all the necessary objects. In this way each $\omega \in \mathcal{Q}$ also has an associated sequence of large scale times before a full return. We write $\hat{p}_{1}=\hat{p}(x)$ and $\hat{p}_{i+1}(x)=\hat{p}_{i}(x)+\hat{p}\left(f^{\hat{p}_{i}(x)}(x)\right)$ so that $\hat{p}_{i+1}(x)$ denotes the total number of iterates making up the first $i+1$ large scale stopping times associated to the point $x$. We have $R(\omega)=\hat{p}_{s}(\omega)+t$ for some $s \geqslant 1, t \leqslant t_{0}$.

We prove two easy but important consequences of the construction.

LEMMA 4.3. - For each $n \geqslant 0$ and each interval $\omega$ on which $\hat{f}^{n}$ is continuous, the distortion of $\hat{f}^{n} \mid \omega$ is uniformly bounded (independently of $\omega$ ).

Proof. - The statement follows directly from the construction and Lemma 4.2. Indeed, the third item of Lemma 4.2 shows that the Koebe space around $f^{\hat{p}(\omega)}$ is at least $\delta^{\prime} / 3$. The additional $t$ iterates do not significantly affect the distortion.

LEMMA 4.4. - For every $i$,

$$
\mid\left\{x ; \hat{p}_{i+1}(x) \text { exists and } \hat{p}_{i+1}>\hat{p}_{i}+k \mid \hat{p}_{i}\right\}\left|\leqslant \frac{3 K}{\delta^{\prime}}\right|\{\hat{p}>k\} \mid .
$$

Here the expression on the left denotes the conditional probability of $\hat{p}_{i+1}>\hat{p}_{i}+k$ on the set of intervals on which $\hat{p}_{i}$ is defined.

Proof. - The statement follows immediately from Lemmas 4.2 and 4.3. Indeed, let $\omega$ be a maximal interval on which $\hat{p}_{i}$ is defined and constant, say $f^{\hat{p}_{i}(\omega)}(\omega)=J \supset \omega_{x}$, where $\omega_{x}$ is as in Lemma 4.2. Let $\omega^{\prime} \subset \omega$ be such that $J^{\prime}=f^{\hat{p}_{i}(\omega)}\left(\omega^{\prime}\right)$ is a component of $J \backslash \omega_{x}$. By construction $\left|J^{\prime}\right| \geqslant \delta^{\prime} / 3$. As the transformation $f^{\hat{p}_{i}(\omega)} \mid \omega$ has distortion bounded by $K=K(\varepsilon)$, we get

$$
\left|\left\{x \in \omega^{\prime} ; \hat{p}_{i+1}(x)>\hat{p}_{i}(x)+k\right\}\right| \leqslant K \frac{\left|\omega^{\prime}\right|}{\left|J^{\prime}\right|}\left|\left\{y \in J^{\prime} ; \hat{p}_{J^{\prime}}(y)>k\right\}\right| .
$$

Because $\left|\left\{\hat{p}_{J^{\prime}}>k\right\}\right| \leqslant|\{\hat{p}>k\}|$ the result follows by summing over all the intervals $\omega^{\prime}$.

\subsection{Bounded distortion}

The function $s$ from (14) is called the separation time function. Notice that $s(x, y)$ is finite for all $x \neq y$, because otherwise $f^{n} \mid(x, y)$ would be homeomorphic for all $n$. The assumptions on $D_{n}$ imply that $\left|\left(f^{n}\right)^{\prime}(x)\right|$ does not converge to 0 for any $x \in X \backslash \bigcup_{n} f^{-n}(\mathcal{C})$, so this cannot happen. By the same token one can show that some iterate of $\hat{f}$ is uniformly expanding, i.e. there exists $N$ such that $\left|\left(\hat{f}^{N}\right)^{\prime}(x)\right| \geqslant 2$ wherever it is defined.

LEMmA 4.5. - There exist $\beta \in(0,1)$ and $C>0$ such that for all $\omega \in \mathcal{Q}$ and all $x, y \in \omega$,

$$
\left|\frac{\hat{f}^{\prime}(x)}{\hat{f}^{\prime}(y)}-1\right| \leqslant C \beta^{s(x, y)} .
$$

Proof. - For small values of $s(x, y)$, (16) follows immediately from Lemma 4.3. Otherwise, uniform expansion of $\hat{f}^{N}$ and Lemma 4.3 imply that $|\hat{f}(x)-\hat{f}(y)| \leqslant\left|\Omega_{0}\right| K 2^{-s(x, y) / N}$. Because the Koebe space around $\hat{f} \mid \omega$ is at least $\delta^{\prime} / 3$, we get 


$$
\begin{aligned}
\left|\frac{\hat{f}^{\prime}(x)}{\hat{f}^{\prime}(y)}-1\right| & \leqslant\left|\left(\frac{\delta^{\prime} / 3|\hat{f}(x)-\hat{f}(y)|+1}{\delta^{\prime} / 3|\hat{f}(x)-\hat{f}(y)|}\right)^{2}-1\right| \\
& \leqslant\left|\left(\frac{\delta^{\prime}+3\left|\Omega_{0}\right| K 2^{-s(x, y) / N}}{\delta^{\prime}}\right)^{2}-1\right| \leqslant C 2^{-s(x, y) / N},
\end{aligned}
$$

where $C=\frac{6 K\left|\Omega_{0}\right|}{\delta^{\prime}}+\left(\frac{3\left|\Omega_{0}\right| K}{\delta^{\prime}}\right)^{2}$. Here we used $K\left(\delta^{\prime} / 3\right)=\left(\frac{1+\delta^{\prime} / 3}{\delta^{\prime} / 3}\right)^{2}$ as Koebe distortion constant, see [19, Chapter IV] in the negative Schwarzian case. In the general case, we take the constant from Theorem B in [24].

\subsection{Return time estimates}

We fix $n \geqslant 1$ and consider the tail $\{R>n\}$ of the return times for $\hat{f}$ on $\Omega_{0}$. Let us agree to use the notation $|\{\hat{p}>n\}|:=\sup \left\{|\{x \in J ; \hat{p}(x)>n\}| /|J| ;|J| \geqslant \delta^{\prime \prime}\right\}$, which was estimated in Proposition 3.1. In the summable case, no explicit estimates were given, except that $\sum_{n}|\{\hat{p}>n\}|<\infty$.

Before starting the proof we introduce some notation. Recall that by construction each $\omega \in \mathcal{Q}$ has an associated sequence

$$
0=\hat{p}_{0}<\hat{p}_{1}<\hat{p}_{2}<\cdots<\hat{p}_{s(\omega)}<R(\omega)
$$

with $R(\omega)=\hat{p}_{s(\omega)}+t$ and clearly $s \leqslant R$. Write $\mathcal{Q}^{(n)}=\{\omega \in \mathcal{Q} ; R(\omega)>n\}$ and let

$$
\mathcal{Q}_{i}^{(n)}=\left\{\omega \in \mathcal{Q}^{(n)} ; \hat{p}_{i-1}<n \leqslant \hat{p}_{i}\right\}
$$

denote the set of elements of $\mathcal{Q}$ with $R(\omega)>n$ and having exactly $i-1$ large scale times before time $n$. Moreover, for each $i$ and every sequence $\left(k_{1}, \ldots, k_{i}\right)$ of positive integers with $\sum k_{j}=n$ we write

$$
\mathcal{Q}_{i}^{(n)}\left(k_{1}, \ldots, k_{i}\right)=\left\{\omega \in \mathcal{Q}_{i}^{(n)} ; k_{j}=\hat{p}_{j}-\hat{p}_{j-1} \text { for } j \leqslant i-1, k_{i}=n-\hat{p}_{i-1}\right\} .
$$

Finally we let

$$
\left|\mathcal{Q}_{i}^{(n)}\right|=\sum_{\omega \in \mathcal{Q}_{i}^{(n)}}|\omega| \text { and }\left|\mathcal{Q}^{(n)}\right|=\sum_{i \leqslant n}\left|\mathcal{Q}_{i}^{(n)}\right| .
$$

Obviously $|\{R>n\}|=\left|\mathcal{Q}^{(n)}\right|$. We are now ready to prove Proposition 4.1.

Proof of Proposition 4.1. - In the stretched exponential case, take $\tilde{\alpha}<\hat{\alpha}<\alpha$, where $\hat{\alpha}$ is as in Proposition 3.1. Both $\tilde{\alpha}$ and $\hat{\alpha}$ can be arbitrarily close to $\alpha$. In the exponential case take $\tilde{\alpha}=\alpha=1$. Let $\eta \in(0,1)$ be a small number to be determined below, depending on $\alpha$ and $\beta$ but not on $n$. We write

$$
|\{R>n\}|=\sum_{i \leqslant n}\left|\mathcal{Q}_{i}\right|=\sum_{i<\eta n^{\tilde{\alpha}}}\left|\mathcal{Q}_{i}\right|+\sum_{\eta n^{\tilde{\alpha} \leqslant i \leqslant n}}\left|\mathcal{Q}_{i}\right| .
$$

Lemma 4.2 says that a fixed proportion $\xi$ of every element in $\mathcal{Q}_{i-1}^{(n)}$ has a full return to $\Omega_{0}$ before its next large scale time. Therefore

$$
\left|\mathcal{Q}_{i}^{(n)}\right| /\left|\mathcal{Q}_{i-1}^{(n)}\right| \leqslant 1-\xi
$$


This implies $\left|\mathcal{Q}_{i}^{(n)}\right| \leqslant(1-\xi)^{i}$ and therefore the second term in (17) satisfies

$$
\sum_{\eta n^{\tilde{\alpha}} \leqslant i \leqslant n}\left|\mathcal{Q}_{i}^{(n)}\right| \leqslant \sum_{\eta n^{\tilde{\alpha}} \leqslant i \leqslant n}(1-\xi)^{i} \leqslant \frac{1}{\xi}(1-\xi)^{\eta n^{\tilde{\alpha}}} .
$$

For the first term write

$$
\sum_{i<\eta n^{\tilde{\alpha}}}\left|\mathcal{Q}_{i}^{(n)}\right|=\sum_{i<\eta n^{\tilde{\alpha}}} \sum_{\substack{\left(k_{1}, \ldots, k_{i}\right) \\ \sum k_{j}=n}}\left|\mathcal{Q}_{i}^{(n)}\left(k_{1}, \ldots, k_{i}\right)\right| .
$$

For a given sequence $\left(k_{1}, \ldots, k_{i}\right)$, Lemma 4.4 and Proposition 3.1 imply

$$
\begin{aligned}
\left|\mathcal{Q}_{i}^{(n)}\left(k_{1}, \ldots, k_{i}\right)\right| & \leqslant\left|\left\{\hat{p}_{i}>\hat{p}_{i-1}+k_{i-1}-1 \mid \hat{p}_{i-1}\right\}\right| \cdots\left|\left\{\hat{p}_{1}>k_{i-1}-1\right\}\right| \\
& \leqslant \widetilde{K}^{i} \prod_{j=1}^{i}\left|\left\{\hat{p}>k_{j}-1\right\}\right| \leqslant \widetilde{K}^{i} \prod_{j=1}^{i} \mathrm{e}^{-\hat{\beta}\left(k_{j}-1\right)^{\hat{\alpha}}} \leqslant\left(\tilde{K} \mathrm{e}^{\hat{\beta}}\right)^{i} \mathrm{e}^{-\hat{\beta} n^{\hat{\alpha}}} .
\end{aligned}
$$

Here $\widetilde{K}=3 K / \delta^{\prime}$ is the constant in the statement of Lemma 4.4. From Lemma 3.4 we have that the number of sequences $\left(k_{1}, \ldots, k_{i}\right)$ as above equals $N_{n, i}^{+}$and satisfies

$$
N_{n, i}^{+} \leqslant \begin{cases}\mathrm{e}^{\hat{\eta} n^{\tilde{\alpha}} \log n} & \text { if } i \leqslant \eta n^{\tilde{\alpha}}, \tilde{\alpha}<1, \\ \mathrm{e}^{\hat{\eta} n} & \text { if } i \leqslant \eta n,\end{cases}
$$

for some $\hat{\eta}=\hat{\eta}(\eta, \tilde{\alpha})$ tending to 0 as $\eta \rightarrow 0$. In the stretched exponential case

$$
\begin{aligned}
\sum_{i<\eta n^{\tilde{\alpha}}}\left|\mathcal{Q}_{i}\right| & =\sum_{i<\eta n^{\tilde{\alpha}}} \sum_{\substack{\left(k_{1}, \ldots, k_{i}\right) \\
\sum_{j} k_{j}=n}}\left|\mathcal{Q}_{i}^{(n)}\left(k_{1}, \ldots, k_{i}\right)\right| \\
& \leqslant \sum_{i<\eta n^{\tilde{\alpha}}} \mathrm{e}^{\hat{\eta} n^{\tilde{\alpha}} \log n}\left(K \mathrm{e}^{\hat{\beta}}\right)^{i} \mathrm{e}^{-\hat{\beta} n^{\hat{\alpha}}} \leqslant \widehat{C} \mathrm{e}^{-\beta^{\prime} n^{\hat{\alpha}}}
\end{aligned}
$$

for some $\widehat{C}, \beta^{\prime}>0$ as long as $\hat{\eta}$ is sufficiently small. In precisely the same way we get $\sum_{i<\eta n}\left|\mathcal{Q}_{i}\right| \leqslant \widehat{C} \mathrm{e}^{-\beta^{\prime} n}$ in the exponential case.

To treat the summable and polynomial case we write $|\{R>n\}|$ as in (17), with $\hat{\alpha}=1$ and $\eta=1 / 2$. The same argument gives an exponential estimate as in (18) for the second term. To estimate the first term, notice that for each $i$ and each sequence $k_{1}, \ldots, k_{i}$ with $\sum k_{j}=n$, the largest $k_{j}$ satisfies $k_{j} \geqslant n / i$. Thus letting

$$
\mathcal{Q}_{i, j}^{(n)}=\left\{\omega \in \mathcal{Q}_{i}^{(n)} ; k_{j^{\prime}}<n / i \text { for } j^{\prime}<j \text { and } k_{j} \geqslant n / i\right\}
$$

we have

$$
\begin{aligned}
\sum_{n \geqslant 1} \sum_{i<n / 2}\left|\mathcal{Q}_{i}^{(n)}\right| & =\sum_{n \geqslant 1} \sum_{i<n / 2} \sum_{j=1}^{i}\left|\mathcal{Q}_{i, j}^{(n)}\right| \\
& \leqslant \sum_{n \geqslant 1} \sum_{i<n / 2} i(1-\xi)^{i-1}|\{\hat{p}>n / i\}| \\
& \leqslant \sum_{i \geqslant 1} i(1-\xi)^{i-1} \sum_{n \geqslant 1}|\{\hat{p}>n / i\}| .
\end{aligned}
$$


Substituting $k=\lfloor n / i\rfloor$, and using the fact that at most $i$ different values of $n$ give the same value of $k$, we find that the above is bounded by $\sum_{i \geqslant 1} i^{2}(1-\xi)^{i-1} \sum_{k \geqslant 1}|\{\hat{p}>k\}|$ which is finite (use Proposition 3.1).

In the polynomial case we get

$$
\sum_{i<n / 2}\left|\mathcal{Q}_{i}^{(n)}\right| \leqslant \mathcal{O}\left(n^{-\alpha}\right) \sum_{i<n / 2} i^{1+\alpha}(1-\xi)^{i}=\mathcal{O}\left(n^{-\alpha}\right)
$$

Together with the exponential estimate for the term $\sum_{i \geqslant n / 2}\left|\mathcal{Q}_{i}^{(n)}\right|$, this yields the proposition.

\subsection{Proof of Theorems 1,2 and 3}

We now state the assumptions and results of Young which we want to apply. Together with the estimates obtained in Proposition 4.1, they easily imply Theorems 1, 2 and 3. Let $m$ denote Lebesgue measure on $X$. L.-S. Young applies the following tower construction for her results. Given a countably piecewise monotone and onto map

$$
\hat{f}: \bigcup_{\omega \in \mathcal{Q}} \omega \rightarrow \Omega_{0}, \quad \hat{f} \mid \omega=f^{R(\omega)}
$$

define a tower

$$
\Omega=\bigsqcup_{\substack{\omega \in \mathcal{Q} \\ 0 \leqslant i<R(\omega)}}(\omega, i)
$$

with an action

$$
g(x, i)= \begin{cases}(x, i+1) & \text { if } x \in \omega, i+1<R(\omega), \\ (\hat{f}(x), 0) & \text { if } x \in \omega, i+1=R(\omega) .\end{cases}
$$

The connection with the original map $f$ is established by means of the projection $\pi(x, i)=f^{i}(x)$. Because $f^{i}$ is smooth and has bounded distortion on each $\omega \in \mathcal{Q}, i<R(\omega)$, this projection has bounded distortion. Also $\pi \circ g=f \circ \pi$. Therefore, if $\nu$ is a $g$-invariant absolutely continuous probability measure on $\Omega, \mu:=\nu \circ \pi^{-1}$ is an invariant absolutely continuous probability measure on the interval.

We summarize Young's results from [32] as far as we need them. For a fixed $\beta \in(0,1)$ as in Lemma 4.5 , let

$$
\mathcal{C}_{\beta}=\left\{\varphi: \Omega \rightarrow \mathbb{R} ; \exists C>0 \forall x, y|\varphi(x)-\varphi(y)| \leqslant C \beta^{s(x, y)}\right\}
$$

and

$$
\mathcal{C}_{\beta}^{+}=\left\{\varphi \in \mathcal{C}_{\beta} ; \varphi \geqslant 0\right\} .
$$

Here we have extended the separation time $s$ to $\Omega$ in the obvious way. Also let $m_{\Omega}$ be Lebesgue measure on $\Omega$. (A priori, $m_{\Omega}$ can be infinite.)

THEOREM (Young [32]). - Suppose that $\hat{f}: \Omega_{0} \rightarrow \Omega_{0}$ is as above, i.e.

$$
m\left(\Omega_{0} \backslash \bigcup_{\omega \in \mathcal{Q}} \omega\right)=0
$$


and (16) holds. Let $\left\{\rho_{n}\right\}$ be a sequence of positive reals related to the tail behaviour of $R$ as follows. If $m(\{R>n\}) \leqslant n^{-\alpha}$, then $\rho_{n}=n^{1-\alpha}$, if $m(\{R>n\}) \leqslant \mathrm{e}^{-\beta n}$, then $\rho_{n}=\mathrm{e}^{-\beta^{\prime} n}$ for some (any) $\beta^{\prime}<\beta$ and if $m(\{R>n\}) \leqslant \mathrm{e}^{-n^{\alpha}}$ for some $\alpha \in(0,1)$, then $\rho_{n}=\mathrm{e}^{-n^{\alpha^{\prime}}}$ for some (any) $\alpha^{\prime}<\alpha$. Then

(1) If $\sum_{n} m(\{R>n\})<\infty$, then $\Omega$ carries a g-invariant absolutely continuous probability measure $\nu$ (Kac's Theorem) and $\frac{d \nu}{d m_{\Omega}} \in \mathcal{C}_{\beta}^{+}$.

(2) For any measure $\tilde{\nu}$ with $\frac{d \tilde{\nu}}{d m_{\Omega}} \in \mathcal{C}_{\beta}^{+}, g_{*}^{n} \tilde{\nu} \rightarrow \nu$ and there exists $C_{\tilde{\nu}}>0$ such that $\left|g_{*}^{n} \tilde{\nu}-\nu\right| \leqslant C_{\tilde{\nu}} \rho_{n}$.

(3) For any pair of functions $\varphi \in L^{\infty}\left(\Omega, m_{\Omega}\right)$ and $\psi \in \mathcal{C}_{\beta}$, there exists $C_{\varphi, \psi}>0$ such that

$$
\left|\int\left(\varphi \circ g^{n}\right) \psi d \nu-\int \varphi d \nu \int \psi d \nu\right| \leqslant C_{\varphi, \psi} \rho_{n}
$$

(4) If $m(\{R>n\}) \leqslant \mathcal{O}\left(n^{-\alpha}\right)$ for some $\alpha>2$, then for any $\varphi \in \mathcal{C}_{\beta}$ which is not a coboundary $(\varphi \neq \psi \circ g-\psi$ for any $\psi)$, the Central Limit Theorem holds, i.e. there exists $\sigma>0$ such that $\frac{1}{\sqrt{n}} \sum_{i=0}^{n-1} \varphi \circ g^{i}$ converges to the normal distribution $\mathcal{N}\left(\int \varphi d \nu, \sigma\right)$.

Remark. - Young states this theorem in terms of a stopping time $\widehat{R}$ which is the extension of $R$ to the entire tower $\Omega$. As it happens

$$
m_{\Omega}(\{\widehat{R}>n\})=\sum_{k \geqslant n} m(\{R>k\}),
$$

so that

$$
m_{\Omega}(\{\widehat{R}>n\}) \leqslant \mathcal{O}\left(n^{-\alpha}\right) \quad \text { if } m(\{R>k\}) \leqslant \mathcal{O}\left(n^{-\alpha-1}\right) .
$$

This explains why the exponent in the polynomial case at first glance looks different from the ones in Young's version.

Using the projection $\pi$, these results immediately carry over to the original map $f$ with measure $\mu=\nu \circ \pi^{-1}$. Using the projection $\pi$, immediately carry over to the original map $f$ with measure $\mu=\nu \circ \pi^{-1}$. With respect to the support of the measure, note that $\hat{f}: \Omega_{0} \rightarrow \Omega_{0}$ is a mixing map, and its invariant measure $\frac{1}{\nu\left(\Omega_{0}\right)} \nu \mid \Omega_{0}$ has the whole interval $\Omega_{0}$ as support. The formula $\mu=\nu \circ \pi^{-1}$ shows that $\Omega_{0} \subset \operatorname{supp}(\mu)$.

Finally, recall from Proposition 4.1 how the tail $m(\{R>n\})$ is related to $d_{n}(c)$. Therefore Theorem 4.4 immediately gives Theorems 1,2 and 3.

\section{Acknowledgements}

The research for this paper was partly supported by the PRODYN program of the European Science Foundation. S. Luzzatto also acknowledges the financial support of EPSRC grant No. GR/K86329.

The suggestions of the referee have considerably improved the present exposition. We acknowledge them gratefully.

\section{REFERENCES}

[1] Baladi V., Viana M., Strong stochastic stability and rate of mixing for unimodal maps, Ann. Sci. Éc. Norm. Sup. 29 (1996) 483-517. 
[2] Benedicks M., Carleson L., On iterations of $x \mapsto 1-a x^{2}$ on $(-1,1)$, Ann. Math. 122 (1985) $1-25$.

[3] Benedicks M., Carleson L., The dynamics of the Hénon map, Ann. Math. 133 (1991) 73-169.

[4] Blokh A., LyUbich M., Measurable dynamics of S-unimodal maps, Ann. Sci. Éc. Norm. Sup. 24 (1991) 545-573.

[5] Bruin H., Luzzatto S., VAn Strien S., Decay of correlation in one-dimensional dynamics, Preprint IHÉS, 1999.

[6] Bruin H., Keller G., Equilibrium states for S-unimodal maps, Ergodic Theory Dynam. Systems 18 (1998) 765-789.

[7] BRUIN H., VAN STRIEN S., Expansion of derivatives in one-dimensional dynamics, Israel J. Math. (to appear).

[8] BRUIN H., VAN STRIEN S., Existence of acips for multimodal maps, in: Global Analysis of Dynamical Systems, Festschrift to Floris Takens for his 60th birthday, IOP Publishing, Bristol, 2001, pp. 433447.

[9] Collet P., Statistics of closes return times for some non uniformly hyperbolic systems, Ergodic Theory Dynam. Systems 21 (2001) 401-420.

[10] GuckenheImer J., Sensitive dependence on initial conditions for unimodal maps, Comm. Math. Phys. 70 (1979) 133-160.

[11] JAKOBSON M.V., Absolutely continuous invariant measures for one-parameter families of onedimensional maps, Comm. Math. Phys. 81 (1981) 39-88.

[12] Keller G., Exponents, attractors, and Hopf decompositions for interval maps, Ergodic Theory Dynam. Systems 10 (1990) 717-744.

[13] Keller G., NOwICKI T., Spectral theory, zeta functions and the distribution of periodic points for Collet-Eckmann maps, Comm. Math. Phys. 149 (1992) 31-69.

[14] KozlovsKi O., Getting rid of the negative Schwarzian derivative condition, Ann. Math. 152 (2000) $743-762$.

[15] LEDRAPPIER F., Some properties of absolutely continuous measures of an interval, Ergodic Theory Dynam. Systems 1 (1981) 77-93.

[16] Liverani C., Saussol B., Vaienti S., A probabilistic approach to intermittency, Ergodic Theory Dynam. Systems 19 (1999) 671-686.

[17] LyUbich M., Milnor J., The Fibonacci unimodal map, J. Amer. Math. Soc. 6 (1993) 425-457.

[18] MAÑÉ R., Hyperbolicity, sinks and measure in one dimensional dynamics, Comm. Math. Phys. 100 (1985) 495-524.

[19] De Melo W., van Strien S., One-Dimensional Dynamics, Springer, 1993.

[20] MisiurewicZ M., Absolutely continuous measures for certain maps of an interval, Publ. IHÉS 53 (1981) $17-51$.

[21] NowiCKI T., SANDS D., Non-uniform hyperbolicity and universal bounds for $S$-unimodal maps, Invent. Math. 132 (1998) 633-680.

[22] NOWICKI T., VAN STRIEN S., Absolutely continuous measures under a summability condition, Invent. Math. 105 (1991) 123-136.

[23] PRZYTYCKI F., Iterations of holomorphic Collet-Eckmann maps: conformal and invariant measures, Trans. Amer. Math. Soc. 350 (1998) 717-742.

[24] VAN STRIEN S., VARGAS E., Real bounds, ergodicity and negative Schwarzian for multimodal maps, Preprint, 2000 and 2001.

[25] Thunberg H., Positive Lyapunov exponents for maps with flat critical points, Ergodic Theory Dynam. Systems (1998) 767-807.

[26] TsuJII M., Small random perturbations of one-dimensional dynamical systems and Margulis-Pesin entropy formula, Random Comput. Dynam. 1 (1992) 59-89.

[27] TsujiI M., Positive Lyapunov exponents in families of one-dimensional dynamical systems, Invent. Math. 111 (1993) 113-137.

[28] Vargas E., Measure of minimal sets of polymodal maps, Ergodic Theory Dynam. Systems 16 (1996) 159-178.

[29] Viana M., Stochastic Dynamics of Deterministic Systems, in: Lecture Notes, Vol. 21, Braz. Math. Colloqium, 1997. 
[30] Young L.-S., Decay of correlations of certain quadratic maps, Comm. Math. Phys. 146 (1992) 123138.

[31] Young L.-S., Statistical properties of dynamical systems with some hyperbolicity, Ann. of Math. 147 (1998) 585-650.

[32] Young L.-S., Recurrence times and rates of mixing, Israel J. Math. 110 (1999) 153-188.

(Manuscrit reçu le 13 mai 2002; accepté, après révision, le 3 juillet 2002.)

\author{
Henk BRUIN \\ Department of Mathematics, \\ University of Groningen, \\ P.O. Box 800, \\ 9700 AV Groningen, \\ The Netherlands \\ E-mail: bruin@math.rug.nl \\ http://www.math.rug.nl/ bruin \\ Stefano LuZZATTO \\ Department of Mathematics, \\ Imperial College, 180 \\ Queen's Gate, \\ London SW7, UK \\ E-mail: stefano.luzzatto@ic.ac.uk \\ http://geometry.ma.ic.ac.uk/ luzzatto \\ Sebastian VAN STRIEN \\ Department of Mathematics, \\ University of Warwick, \\ Coventry CV4 7AL, UK \\ E-mail: strien@maths.warwick.ac.uk \\ http://www.maths.warwick.ac.uk/ strien
}

